Discrete Comput Geom 28:175-199 (2002)

DOI: $10.1007 / \mathrm{s} 00454-002-2759-7$

\title{
Lattice Points, Dedekind Sums, and Ehrhart Polynomials of Lattice Polyhedra*
}

\author{
Beifang Chen \\ Department of Mathematics, \\ Hong Kong University of Science and Technology, \\ Clear Water Bay, Kowloon, Hong Kong \\ mabfchen@ust.hk
}

Dedicated to the Memory of Gian-Carlo Rota

\begin{abstract}
Let $\sigma$ be a simplex of $\mathbf{R}^{N}$ with vertices in the integral lattice $\mathbf{Z}^{N}$. The number of lattice points of $m \sigma(=\{m \alpha: \alpha \in \sigma\})$ is a polynomial function $L(\sigma, m)$ of $m \geq 0$. In this paper we present: (i) a formula for the coefficients of the polynomial $L(\sigma, t)$ in terms of the elementary symmetric functions; (ii) a hyperbolic cotangent expression for the generating functions of the sequence $L(\sigma, m), m \geq 0$; (iii) an explicit formula for the coefficients of the polynomial $L(\sigma, t)$ in terms of torsion. As an application of (i), the coefficient for the lattice $n$-simplex of $\mathbf{R}^{n}$ with the vertices $\left(0, \ldots, 0, a_{j}, 0, \ldots, 0\right)(1 \leq j \leq n)$ plus the origin is explicitly expressed in terms of Dedekind sums; and when $n=2$, it reduces to the reciprocity law about Dedekind sums. The whole exposition is elementary and self-contained.
\end{abstract}

\section{Introduction}

Let $P$ be a lattice polytope of $\mathbf{R}^{N}$, i.e., the vertices of $P$ are points of the integral lattice $\mathbf{Z}^{N}$. The dilation of $P$ by a positive integer $m$ is the polytope $m P=\{m \alpha: \alpha \in P\}$; the relative interior of $P$ is the set $P^{0}=P-\partial P$. Let $L(P, m)$ and $L\left(P^{0}, m\right)$ denote the number of lattice points of $m P$ and $m P^{0}$, respectively. It is well known that $L(P, m)$ and $L\left(P^{0}, m\right)$ are polynomial functions of $m$, called the Ehrhart polynomial, and satisfy the functional equation

$$
L(P,-t)=(-1)^{\operatorname{dim} P} L\left(P^{0}, t\right)
$$

* This research was supported by RGC Competitive Earmarked Research Grants HKUST6119/00P. 
called the reciprocity law by Ehrhart [9]. Let $n=\operatorname{dim} P$ and write

$$
L(P, t)=\sum_{j=0}^{n} c_{j}(P) t^{j}
$$

It is known that $c_{n}(P)$ is the volume vol $P, c_{n-1}(P)$ is the boundary surface area vol $\partial P$, and $c_{0}(P)$ is the (combinatorial) Euler characteristic $\chi(P)$, where vol is normalized by the determinants of the sublattices induced by the corresponding faces of $P$; see [20]. The Ehrhart polynomial $L(P, m)$ can be defined for any bounded lattice polyhedron $P$ (a union of finitely many relatively open lattice polytopes). If $P$ is not compact, the combinatorial Euler characteristic is usually different from that defined by the alternating sum of Betti numbers; while if $P$ is compact, they are the same; see [4] and [12] for example. The other coefficients of Ehrhart polynomials are still mysterious, even for a general lattice 3-simplex, until the recent work of Morelli [16] in $\mathbf{R}^{n}$, Pommersheim [18] in $\mathbf{R}^{3}$, Kantor and Khovanskii [11] in $\mathbf{R}^{4}$, Cappell and Shaneson [3] in $\mathbf{R}^{n}$, Brion and Vergne [2] in $\mathbf{R}^{n}$, and Diaz and Robins [8] in $\mathbf{R}^{n}$. For instance, the coefficients for a lattice tetrahedron of $\mathbf{R}^{3}$ with vertices $(0,0,0),(a, 0,0),(0, b, 0),(0,0, c)$ are completely determined; Kanor and Khovanskii [11] gave a complete description of the codimension 2 coefficients of the Ehrhart polynomials. However, the work of [16] used the Bott theorem of differential geometry to express these coefficients in terms of rational functions on Grassmanians; [3] and [11] used the Riemann-Roch theorem of algebraic geometry to relate the Todd class and the Chern class of toric variety to express these coefficients in terms of Dedekind sums and cotangent functions; and the work of [2] used the technique of deformation for simple-polytopes and a combinatorial version of the Riemann-Roch theorem. The work of [8] is to express the generating functions of the sequences $L(\sigma, m)$ and $L\left(\sigma^{0}, m\right)$ in terms of hyperbolic cotangent functions for a lattice simplex $\sigma$, whose vertices are the column vectors of a lower triangular matrix plus the origin. These generating functions are important because the coefficients of Ehrhart polynomials can be easily computed from their explicit expressions. The method of [8] is analytic, using the Poisson summation and the Fourier analysis, and involves some lengthy estimations. It seems that it is still an open problem to find a general formula with geometric interpretation for the coefficients of Ehrhart polynomials.

This paper is inspired by the work of Brion and Vergne [2], Diaz and Robins [8], and the original work of Ehrhart [9]. We present in a simple direct way: (i) a formula for the coefficients of Ehrhart polynomials in terms of the elementary symmetric functions; (ii) a hyperbolic cotangent expression for the generating functions of the sequences $L(\sigma, m)$ and $L\left(\sigma^{0}, m\right)$ of any lattice simplex $\sigma$; (iii) an explicit formula for the coefficients of Ehrhart polynomials in terms of torsion. The whole exposition is elementary and selfcontained.

To state our results, we begin with some notations. Let $\sigma$ be an $n$-simplex of $\mathbf{R}^{N}$ with vertices $\alpha_{0}, \alpha_{1}, \ldots, \alpha_{n}$ in $\mathbf{Z}^{N}$; its relative interior is denoted by $\sigma^{0}=\sigma-\partial \sigma$. We define the determined set of $\sigma$ at the vertex $\alpha_{0}$ by

$$
D\left(\sigma, \alpha_{0}\right)=\left\{\sum_{j=1}^{n} u_{j}\left(\alpha_{j}-\alpha_{0}\right) \in \mathbf{Z}^{N}: 0 \leq u_{j}<1\right\} .
$$


For each vector $\gamma=\sum_{j=1}^{n} u_{j}\left(\alpha_{j}-\alpha_{0}\right) \in D\left(\sigma, \alpha_{0}\right)$, we write $|\gamma|=\sum_{j=1}^{n} u_{j}$. Our first main result is on the $j$ th coefficients of $L\left(\sigma^{0}, t\right)$ and $L(\sigma, t)$ :

$$
\begin{aligned}
c_{j}\left(\sigma^{0}\right) & =\frac{1}{n !} \sum_{\gamma \in D\left(\sigma, \alpha_{0}\right)} s_{n-j}(\lceil|\gamma|\rceil-1, \ldots,\lceil|\gamma|\rceil-n), \\
c_{j}(\sigma) & =\frac{1}{n !} \sum_{\gamma \in D\left(\sigma, \alpha_{0}\right)} s_{n-j}(1-\lceil|\gamma|\rceil, \ldots, n-\lceil|\gamma|\rceil),
\end{aligned}
$$

where $s_{n-j}\left(x_{1}, \ldots, x_{n}\right)$ is the $(n-j)$ th elementary symmetric function of $n$ variables; $\lceil|\gamma|\rceil$ is the smallest integer greater than or equal to $|\gamma|$. Now the reciprocity law is read to

$$
c_{j}(\sigma)=(-1)^{n-j} c_{j}\left(\sigma^{0}\right) .
$$

The coefficients $c_{j}$ are obviously well-defined for any bounded lattice polyhedra; thus $c_{j}$ are unimodular invariant measures on bounded lattice polyhedra of $\mathbf{R}^{N}$. Coincidently, the measures $c_{j}$ are discrete analogs of the intrinsic volumes $V_{j}$, which are unique rigid motion invariant measures on bounded polyhedra of $\mathbf{R}^{N}$, such that

$$
V_{j}(P)=s_{j}\left(x_{1}, \ldots, x_{n}\right)
$$

for rectangular parallelotopes $P$ of side length $x_{1}, \ldots, x_{n}$; see [12].

Let $A$ be the $N \times n$ matrix whose columns are the vectors $\alpha_{1}-\alpha_{0}, \ldots, \alpha_{n}-\alpha_{0}$. The matrix $A$ can be viewed as an Abelian group homomorphism from $\mathbf{Z}^{n}$ to $\mathbf{Z}^{N}$; the torsion of $A$ is defined to be the order of the torsion subgroup of $\mathbf{Z}^{N} / \operatorname{Im} A$, denoted tor $A$. Set $p=$ tor $A$; our second main result is

$$
\begin{array}{r}
c_{j}\left(\sigma^{0}\right)=\frac{1}{n !} \sum_{\substack{0 \leq h_{1}, \ldots, h_{n} \leq p-1 \\
h=\left(h_{1}, \ldots, h_{n}\right) \in \mathbf{Z}^{n} \\
p \mid A h}} s_{n-j}\left(\left\lceil\frac{h_{1}+\cdots+h_{n}}{p}\right\rceil-1,\right. \\
\left.\ldots,\left\lceil\frac{h_{1}+\cdots+h_{n}}{p}\right\rceil-n\right),
\end{array}
$$

where $p \mid A h$ means that $p$ divides each entry of the vector $A h$.

The matrix $A$ can be transformed into an upper triangular form (7), having $a_{j j}>0$, by the modified Gaussian elimination (adding an integral multiple of one row to another row; switching two rows):

$$
\left(\begin{array}{cccc}
a_{11} & a_{12} & \cdots & a_{1 n} \\
0 & a_{22} & \cdots & a_{2 n} \\
\vdots & \vdots & & \vdots \\
0 & 0 & \cdots & a_{n n} \\
0 & 0 & \cdots & 0 \\
\vdots & \vdots & & \vdots \\
0 & 0 & \cdots & 0
\end{array}\right) .
$$


When $N=n$, this upper triangular matrix is known as the Hermite normal form; see [17]. For $\left(j_{1}, \ldots, j_{n}\right) \in \prod_{i=1}^{n}\left[0, a_{i i}\right) \cap \mathbf{Z}$, we define $u\left(j_{1}, \ldots, j_{n}\right)=\left(u_{1}, \ldots, u_{n}\right)$ inductively by

$$
\left\{\begin{aligned}
u_{n}= & \frac{j_{n}}{a_{n n}}, \\
u_{i}= & \frac{\left\lceil a_{i(i+1)} u_{i+1}+\cdots+a_{i n} u_{n}\right\rceil}{a_{i i}} \\
& -\frac{a_{i(i+1)} u_{i+1}+\cdots+a_{i n} u_{n}}{a_{i i}}+\frac{j_{i}}{a_{i i}}, \quad 1 \leq i \leq n-1 ;
\end{aligned}\right.
$$

and $\left|u\left(j_{1}, \ldots, j_{n}\right)\right|=u_{1}+\cdots+u_{n}$. Then (4) and (5) for $c_{j}\left(\sigma^{0}\right)$ and $c_{j}(\sigma)$ can be further written as

$$
\begin{aligned}
c_{j}\left(\sigma^{0}\right) & =\frac{1}{n !} \sum_{j_{1}=0}^{a_{11}-1} \cdots \sum_{j_{n}=0}^{a_{n n}-1} s_{n-j}\left(\left\lceil\left|u\left(j_{1}, \ldots, j_{n}\right)\right|\right\rceil-1, \ldots,\left\lceil\left|u\left(j_{1}, \ldots, j_{n}\right)\right|\right\rceil-n\right), \\
c_{j}(\sigma) & =\frac{1}{n !} \sum_{j_{1}=0}^{a_{11}-1} \cdots \sum_{j_{n}=0}^{a_{n n}-1} s_{n-j}\left(1-\left\lceil\left|u\left(j_{1}, \ldots, j_{n}\right)\right|\right\rceil, \ldots, n-\left\lceil\left|u\left(j_{1}, \ldots, j_{n}\right)\right|\right\rceil\right) .
\end{aligned}
$$

Consequently, the generating functions of the sequences $L(\sigma, m)$ and $L\left(\sigma^{0}, m\right)$ are given by

$$
\begin{aligned}
\sum_{m=0}^{\infty} L(\sigma, m) t^{m} & =\frac{1}{(1-t)^{n+1}} \sum_{j_{1}=0}^{a_{11}-1} \cdots \sum_{j_{n}=0}^{a_{n n}-1} t^{\left\lceil\left|u\left(j_{1}, \ldots, j_{n}\right)\right|\right\rceil} \\
\sum_{m=1}^{\infty} L\left(\sigma^{0}, m\right) t^{m} & =\frac{t^{n+1}}{(1-t)^{n+1}} \sum_{j_{1}=0}^{a_{11}-1} \cdots \sum_{j_{n}=0}^{a_{n n}-1} t^{-\left\lceil\left|u\left(j_{1}, \ldots, j_{n}\right)\right|\right\rceil} .
\end{aligned}
$$

If the matrix $A$ is already in upper triangular form (7) without applying the modified Gaussian elimination, then the map $\tilde{u}:\left(j_{1}, \ldots, j_{n}\right) \mapsto \sum_{i=1}^{n} u_{i}\left(\alpha_{i}-\alpha_{0}\right)$ by (8) is a one-to-one correspondence between $\prod_{i=1}^{n}\left[0, a_{i i}\right) \cap \mathbf{Z}$ and the determined set $D\left(\sigma, \alpha_{0}\right)$, which gives an efficient algorithm to list all elements of $D\left(\sigma, \alpha_{0}\right)$.

Let $B$ be the $(N+1) \times(n+1)$ matrix whose columns are the vectors $\beta_{0}=\left(1, \alpha_{0}\right)$, $\beta_{1}=\left(1, \alpha_{1}\right), \ldots, \beta_{n}=\left(1, \alpha_{n}\right)$. Let $\operatorname{adj}\left(B^{\mathrm{T}} B\right)$ denote the adjoint matrix of $B^{\mathrm{T}} B$. For $0 \leq j \leq n$ and $0 \leq k \leq N$, define positive integers:

$$
\begin{aligned}
a_{j} & =\operatorname{gcd} \text { of the } j \text { th } \operatorname{row} \text { of } \operatorname{adj}\left(B^{\mathrm{T}} B\right) B^{\mathrm{T}}, \\
b_{k} & =\operatorname{gcd} \text { of the } k \text { th row of } B \operatorname{diag}\left[a_{0}, a_{1}, \ldots, a_{n}\right], \\
p_{j} & =\frac{\left|\operatorname{det} B^{\mathrm{T}} B\right|}{a_{j}}, \\
q_{k} & =\frac{\left|\operatorname{det} B^{\mathrm{T}} B\right|}{\operatorname{gcd}\left(b_{k}, \operatorname{det} B^{\mathrm{T}} B\right)} .
\end{aligned}
$$


Set $G=\prod_{k=0}^{N} \mathbf{Z} / q_{k} \mathbf{Z}$; our third main result is on the generating functions of the sequences $L(\sigma, m)$ and $L\left(\sigma^{0}, m\right)$ :

$$
\begin{aligned}
\sum_{m=0}^{\infty} L(\sigma, m) e^{-2 \pi m s} & =\frac{1}{2^{n+1}|G|} \sum_{g \in G} \prod_{j=0}^{n}\left(1+\operatorname{coth} \frac{\pi}{p_{j}}\left(s+\imath\left\langle\beta_{j}, g\right\rangle\right)\right) ; \\
\sum_{m=1}^{\infty} L\left(\sigma^{0}, m\right) e^{-2 \pi m s} & =\frac{(-1)^{n+1}}{2^{n+1}|G|} \sum_{g \in G} \prod_{j=0}^{n}\left(1-\operatorname{coth} \frac{\pi}{p_{j}}\left(s+\imath\left\langle\beta_{j}, g\right\rangle\right)\right) .
\end{aligned}
$$

Set $p=$ tor $A$; we further have

$$
\begin{aligned}
& \sum_{m=0}^{\infty} L(\sigma, m) e^{-2 \pi m s} \\
& =\frac{1}{2^{n+1} p^{N+1}} \sum_{\substack{h_{0}, h_{1}, \ldots, h_{N}=0 \\
h=\left(h_{0}, h_{1}, \ldots, h_{N}\right)}}^{p-1} \prod_{j=0}^{n}\left(1+\operatorname{coth} \frac{\pi}{p}\left(s+\imath\left\langle\beta_{j}, h\right\rangle\right)\right) ; \\
& \sum_{m=1}^{\infty} L\left(\sigma^{0}, m\right) e^{-2 \pi m s} \\
& =\frac{(-1)^{(n+1)}}{2^{n+1} p^{N+1}} \sum_{\substack{h_{0}, h_{1}, \ldots, h_{N}=0 \\
h=\left(h_{0}, h_{1}, \ldots, h_{N}\right)}}^{p-1} \prod_{j=0}^{n}\left(1-\operatorname{coth} \frac{\pi}{p}\left(s+\imath\left\langle\beta_{j}, h\right\rangle\right)\right) .
\end{aligned}
$$

In particular, when $N=n$, one can choose

$$
\begin{aligned}
a_{j} & =\operatorname{gcd} \text { of the } j \text { th row of adj } B, \\
b_{k} & =\operatorname{gcd} \text { of the } k \text { th row of } B \operatorname{diag}\left[a_{0}, a_{1}, \ldots, a_{n}\right], \\
p_{j} & =\frac{|\operatorname{det} B|}{a_{j}}, \\
q_{k} & =\frac{|\operatorname{det} B|}{\operatorname{gcd}\left(b_{k}, \operatorname{det} B\right)}
\end{aligned}
$$

for (13) and (14). Moreover, in this special case $N=n$, if the $n \times n$ matrix $A$ is in upper triangular form (7), one can simply choose $p_{j}=q_{j}=a_{j j} \cdots a_{n n}, 0 \leq j \leq n$, where $a_{00}=1 ; G=\prod_{j=0}^{n} \mathbf{Z} / p_{j} \mathbf{Z}$; and recover the main result of [8].

The hyperbolic cotangent functions can be also written as cotangent functions. A notable feature of our formulas is that the group $G$ can be selected as long as certain conditions are satisfied. Since every bounded lattice polyhedron $P$ can be decomposed into a disjoint union of relatively open lattice simplices, the coefficients of the Ehrhart polynomial $L(P, t)$ can be computed by the coefficient formulas (4)-(6), (9), (10) and the generating functions (11)-(16).

As an application of the coefficient formula (4), we consider the lattice $n$-simplex $\sigma\left(a_{1}, \ldots, a_{n}\right)$ with the vertices $\left(0, \ldots, 0, a_{j}, 0, \ldots, 0\right)(1 \leq j \leq n)$ plus the origin; and express the coefficient $c_{n-2}$ explicitly in terms of Dedekind sums. When $n=2$, 
it reduces to the reciprocity law about Dedekind sums. The other coefficients can be similarly expressed in terms of Zagier's higher-dimensional Dedekind sums. While for an arbitrary lattice simplex, it is not clear whether the higher Dedekind sums are enough to express those coefficients. For this reason, we introduce a matrix Dedekind sum, which seems to be enough to do the job; and it should be of interest to study itself.

\section{Deriving Ehrhart Polynomials}

To derive our first result and to make our exposition transparent and self-contained, we essentially follow the idea of Ehrhart [9] to derive some known (but not well-known) results, with more sophisticated notations and arguments.

Let $\Lambda$ be an $N$-dimensional lattice of $\mathbf{R}^{N}$. Let $\sigma$ be an $n$-simplex with vertices $\alpha_{0}, \alpha_{1}, \ldots, \alpha_{n}$. We assume that there is a positive real number $d$ such that all $d \alpha_{j}$ are points of $\Lambda$. Choose a lattice basis of $\Lambda$; the vertices of $\sigma$ may have irrational coordinates with respect to the lattice basis, and in this case $d$ must be an irrational number. If the vertices of $\sigma$ have rational coordinates with respect to the lattice basis, then $d$ can be the $\mathrm{lcm}$ (least common multiple) of the denominators of the rational vertices. We want to count the number of points of $\Lambda$ in the dilation $\lambda \sigma$ by a positive real number $\lambda$. Let $E(\sigma, \Lambda, \lambda)$ denote the number of points of $\lambda \sigma \cap \Lambda$, and $E\left(\sigma^{0}, \Lambda, \lambda\right)$ the number of points of $\lambda \sigma^{0} \cap \Lambda$. Write each $\alpha \in \lambda \sigma$ and $\beta \in \lambda \sigma^{0}$ as the unique affine linear combinations:

$$
\begin{array}{rlrl}
\alpha=\sum_{j=0}^{n} a_{j} \lambda \alpha_{j}, & & \sum_{j=0}^{n} a_{j}=1, & a_{j} \geq 0 ; \\
\beta=\sum_{j=0}^{n} b_{j} \lambda \alpha_{j}, & \sum_{j=0}^{n} b_{j}=1, & b_{j}>0 .
\end{array}
$$

By the division algorithm, $\alpha$ and $\beta$ can be further uniquely written as

$$
\begin{aligned}
& \alpha=\sum_{j=0}^{n} u_{j} \alpha_{j}+\sum_{j=0}^{n} k_{j} d \alpha_{j}, \\
& \beta=\sum_{j=0}^{n} v_{j} \alpha_{j}+\sum_{j=0}^{n} l_{j} d \alpha_{j},
\end{aligned}
$$

with real numbers $u_{j}$ and $v_{j}$, non-negative integers $k_{j}$ and $l_{j}$, such that

$$
\begin{array}{lll}
\sum_{j=0}^{n} u_{j}+d \sum_{j=0}^{n} k_{j}=\lambda, & 0 \leq u_{j}<d, & k_{j}=\left\lfloor\frac{a_{j} \lambda}{d}\right\rfloor, \\
\sum_{j=0}^{n} v_{j}+d \sum_{j=0}^{n} l_{j}=\lambda, & 0<v_{j} \leq d, & l_{j}=\left\lceil\frac{b_{j} \lambda}{d}\right\rceil-1 .
\end{array}
$$

Clearly, $\alpha$ is a point of $\Lambda$ if and only if $\gamma=\sum_{j=0}^{n} u_{j} \alpha_{j}$ is a point of $\Lambda ; \beta$ is a point of $\Lambda$ if and only if $\sum_{j=0}^{n} v_{j} \alpha_{j}$ is a point of $\Lambda$. Write $|\gamma|=\sum_{j=0}^{n} u_{j}$ and $k=\sum_{j=0}^{n} k_{j}$. For 
a non-negative real number $\ell \leq(n+1) d$, define the determined sets of $\sigma$ at level $\ell$ :

$$
\begin{aligned}
& D(\sigma, \Lambda, \ell)=\left\{\gamma=\sum_{j=0}^{n} u_{j} \alpha_{j} \in \Lambda: 0 \leq u_{j}<d,|\gamma|=\ell\right\} ; \\
& \bar{D}(\sigma, \Lambda, \ell)=\left\{\gamma=\sum_{j=0}^{n} u_{j} \alpha_{j} \in \Lambda: 0<u_{j} \leq d,|\gamma|=\ell\right\} .
\end{aligned}
$$

For each $\gamma \in D(\sigma, \Lambda, \ell)$, since $0 \leq|\gamma|<(n+1) d$, the integer $k=(\lambda-|\gamma|) / d$ belongs to the half-open and half-closed interval $(\lambda / d-n-1, \lambda / d]$; while for each $\gamma \in \bar{D}(\sigma, \Lambda, \ell)$, since $0<|\gamma| \leq(n+1) d$, the integer $k=(\lambda-|\gamma|) / d$ belongs to the interval $[\lambda / d-n-1, \lambda / d)$. Notice that when $|\gamma|=\lambda-d k$ is fixed, the number of tuples $\left(k_{0}, k_{1}, \ldots, k_{n}\right)$, with non-negative integer entries such that $\sum_{j=0}^{n} k_{j}=k$, is the binomial coefficient $\left(\begin{array}{c}k+n \\ n\end{array}\right)$; this is true even when $k$ is a negative integer. We thus have the following proposition.

Proposition 2.1. Let $\sigma$ be an n-simplex whose vertices can be dilated into points of $\Lambda$ by a single positive real number $d$. Then for any positive real number $\lambda$,

$$
\begin{aligned}
E(\sigma, \Lambda, \lambda) & =\sum_{k \in(\lambda / d-n-1, \lambda / d] \cap \mathbf{Z}}|D(\sigma, \Lambda, \lambda-d k)|\left(\begin{array}{c}
k+n \\
n
\end{array}\right) ; \\
E\left(\sigma^{0}, \Lambda, \lambda\right) & =\sum_{k \in[\lambda / d-n-1, \lambda / d) \cap \mathbf{Z}}|\bar{D}(\sigma, \Lambda, \lambda-d k)|\left(\begin{array}{c}
k+n \\
n
\end{array}\right) .
\end{aligned}
$$

Notice that the right side of (19) and (20) are well-defined for non-positive real numbers $\lambda$. From now on we define $E(\sigma, \Lambda, \lambda)$ and $E\left(\sigma^{0}, \Lambda, \lambda\right)$ for all real numbers $\lambda$ by (19) and (20), respectively.

Let $r$ and $s$ be non-negative integers such that $r+s=(n+1) d$. There is a one-toone correspondence between $D(\sigma, \Lambda, r)$ and $\bar{D}(\sigma, \Lambda, s)$, sending the affine coordinates $\left(u_{0}, u_{1}, \ldots, u_{n}\right)$ to $\left(d-u_{0}, d-u_{1}, \ldots, d-u_{n}\right)$. This is well-defined because $\sum_{j=0}^{n} u_{j} \alpha_{j}$ is a point of $\Lambda$ if and only if $\sum_{j=0}^{n}\left(d-u_{j}\right) \alpha_{j}$ is a point of $\Lambda$. Write $s=\lambda-d k$ in (20), then $\bar{D}(\sigma, \Lambda, \lambda-d k)$ can be replaced by $D(\sigma, \Lambda, r)$ with $r=(n+1) d-s=(n+1) d-\lambda+d k$. Thus

$$
E\left(\sigma^{0}, \Lambda, \lambda\right)=\sum_{k \in[\lambda / d-n-1, \lambda / d) \cap \mathbf{Z}}|D(\sigma, \Lambda,(n+1) d-\lambda+d k)|\left(\begin{array}{c}
k+n \\
n
\end{array}\right) .
$$

Set $(n+1) d-\lambda+d k=-\lambda-d l$, we have $k=-l-n-1 ; l$ is an integer if and only if $k$ is an integer; $-\lambda / d-n-1<l \leq-\lambda / d$. Therefore

$$
\begin{aligned}
E\left(\sigma^{0}, \Lambda, \lambda\right) & =\sum_{l \in(-\lambda / d-n-1,-\lambda / d] \cap \mathbf{Z}}|D(\sigma, \Lambda,-\lambda-d l)|\left(\begin{array}{c}
-l-1 \\
n
\end{array}\right) \\
& =\sum_{l \in(-\lambda / d-n-1,-\lambda / d] \cap \mathbf{Z}}|D(\sigma, \Lambda,-\lambda-d l)|(-1)^{n}\left(\begin{array}{c}
l+n \\
n
\end{array}\right) .
\end{aligned}
$$


In the last step we have made use of the reciprocity property of binomial coefficients:

$$
\left(\begin{array}{c}
n+l \\
n
\end{array}\right)=(-1)^{n}\left(\begin{array}{c}
-l-1 \\
n
\end{array}\right) .
$$

We state this result in the following as a reciprocity law.

Proposition 2.2. Let $\sigma$ be an n-simplex whose vertices can be transformed into points of $\Lambda$ multiplied by a single positive real number $d$. Then for any real number $\lambda$,

$$
E\left(\sigma^{0}, \Lambda,-\lambda\right)=(-1)^{n} E(\sigma, \Lambda, \lambda) .
$$

The reciprocity law (21) has been generalized to arbitrary rational polyhedra and rational polyhedral functions in [5] by using weight and boundary weight functions. The idea there can be further applied to get reciprocity laws for irrational polyhedra and polyhedral weight functions.

Consider the special case where $\sigma$ is a $\Lambda$-lattice simplex; $d=1$. If we restrict $\lambda$ to integers $m$, then $E(\sigma, \Lambda, m)$ and $E\left(\sigma^{0}, \Lambda, m\right)$ are polynomial functions of integral variable $m$, called Ehrhart polynomials. We denote these polynomials by $L(\sigma, \Lambda, m)$ and $L\left(\sigma^{0}, \Lambda, m\right)$, respectively. Notice that when $m$ is replaced by non-integral real numbers $\lambda$ in $L(\sigma, \Lambda, m)$, the real-valued function $L(\sigma, \Lambda, \lambda)$ is different from $E(\sigma, \Lambda, \lambda)$. The function $E(\sigma, \Lambda, \lambda)$ is always integer-valued, while $L(\sigma, \Lambda, \lambda)$ is obviously not. We write $L(\sigma, \Lambda, m)$ and $L\left(\sigma^{0}, \Lambda, m\right)$ more explicitly in the following proposition by changing the summation indices of (19) and (20).

Proposition 2.3. Let $\sigma$ be an $n$-simplex with vertices in $\Lambda$. Then the polynomial functions $L(\sigma, \Lambda, m)$ and $L\left(\sigma^{0}, \Lambda, m\right)$ are given by

$$
\begin{aligned}
L(\sigma, \Lambda, m) & =\sum_{k=0}^{n}|D(\sigma, \Lambda, k)|\left(\begin{array}{c}
m+n-k \\
n
\end{array}\right) ; \\
L\left(\sigma^{0}, \Lambda, m\right) & =\sum_{k=0}^{n}|D(\sigma, \Lambda, k)|\left(\begin{array}{c}
m+k-1 \\
n
\end{array}\right) .
\end{aligned}
$$

Let $s_{j}$ denote the $j$ th elementary symmetric function of $n$ variables, i.e.,

$$
s_{j}\left(x_{1}, x_{2}, \ldots, x_{n}\right)=\sum_{k_{1}<k_{2}<\cdots<k_{j}} x_{k_{1}} x_{k_{2}} \cdots x_{k_{j}} .
$$

With convention $s_{0} \equiv 1$, the binomial coefficient in (23) can be written as

$$
\begin{aligned}
\left(\begin{array}{c}
m+k-1 \\
n
\end{array}\right) & =\frac{1}{n !}(m+k-1)(n+k-2) \cdots(m+k-n) \\
& =\frac{1}{n !} \sum_{j=0}^{n} m^{j} s_{n-j}(k-1, k-2, \ldots, k-n) .
\end{aligned}
$$


This immediately gives rise to the following simple formulas on the coefficients of Ehrhart polynomials. Formulas (22) and (23) were first obtained by Ehrhart [9], then reproved by Macdonald [13], [14], and appeared in Danilov's treatise [7] on toric varieties. It seems that the following coefficient formulas in elementary symmetric functions were overlooked, until it was recently observed in [6].

Theorem 2.4. Let $\sigma$ be an $n$-simplex with vertices in $\Lambda$. Then the $j$ th coefficients of $L(\sigma, \Lambda, t)$ and $L\left(\sigma^{0}, \Lambda, t\right)$ are given by

$$
\begin{aligned}
c_{j}\left(\sigma^{0}, \Lambda\right) & =\frac{1}{n !} \sum_{k=0}^{n}|D(\sigma, \Lambda, k)| s_{n-j}(k-1, \ldots, k-n) ; \\
c_{j}(\sigma, \Lambda) & =\frac{1}{n !} \sum_{k=0}^{n}|D(\sigma, \Lambda, k)| s_{n-j}(1-k, \ldots, n-k) .
\end{aligned}
$$

Since counting lattice points is invariant under integral translations, the number of lattice points in $m \sigma$ is the same as the number of lattice points in the dilation $m \sigma^{\prime}$, where $\sigma^{\prime}$ is the simplex with the vertices $\alpha_{1}-\alpha_{0}, \ldots, \alpha_{n}-\alpha_{0}$ and the origin. Then every $\alpha \in m \sigma^{\prime}$ and $\beta \in m \sigma^{\prime 0}$ can be uniquely written as the linear combinations:

$$
\begin{aligned}
\alpha=\sum_{j=1}^{n} a_{j}\left(\alpha_{j}-\alpha_{0}\right), & & \sum_{j=1}^{n} a_{j} \leq m, & a_{j} \geq 0 ; \\
\beta=\sum_{j=1}^{n} b_{j}\left(\alpha_{j}-\alpha_{0}\right), & & \sum_{j=1}^{n} b_{j}<m, & b_{j}>0 .
\end{aligned}
$$

Similarly, $\alpha$ and $\beta$ can be further uniquely written as

$$
\begin{gathered}
\alpha=\sum_{j=1}^{n} u_{j}\left(\alpha_{j}-\alpha_{0}\right)+\sum_{j=1}^{n} k_{j}\left(\alpha_{j}-\alpha_{0}\right), \\
\beta=\sum_{j=1}^{n} v_{j}\left(\alpha_{j}-\alpha_{0}\right)+\sum_{j=1}^{n} l_{j}\left(\alpha_{j}-\alpha_{0}\right),
\end{gathered}
$$

with real numbers $u_{j}$ and $v_{j}$, non-negative integers $k_{j}$ and $l_{j}$, such that

$$
\begin{array}{ll}
\sum_{j=1}^{n} u_{j}+\sum_{i=1}^{n} k_{j} \leq m, & 0 \leq u_{j}<1 ; \\
\sum_{j=1}^{n} v_{j}+\sum_{j=1}^{n} l_{j}<m, & 0<v_{j} \leq 1 .
\end{array}
$$

Moreover, $\alpha$ and $\beta$ are points of $\Lambda$ if and only if $\sum_{j=1}^{n} u_{j}\left(\alpha_{j}-\alpha_{0}\right)$ and $\sum_{j=1}^{n} v_{j}\left(\alpha_{j}-\alpha_{0}\right)$ are points of $\Lambda$, respectively. Define the determined sets:

$$
\begin{aligned}
& D\left(\sigma, \alpha_{0} ; \Lambda\right)=\left\{\sum_{j=1}^{n} u_{j}\left(\alpha_{j}-\alpha_{0}\right) \in \Lambda: 0 \leq u_{j}<1\right\} ; \\
& \bar{D}\left(\sigma, \alpha_{0} ; \Lambda\right)=\left\{\sum_{j=1}^{n} v_{j}\left(\alpha_{j}-\alpha_{0}\right) \in \Lambda: 0<v_{j} \leq 1\right\} .
\end{aligned}
$$


For each $\gamma=\sum_{j=1}^{n} u_{j}\left(\alpha_{j}-\alpha_{0}\right)$ of $D(\sigma, \Lambda) \cup \bar{D}(\sigma, \Lambda)$, we write $|\gamma|=\sum_{j=1}^{n} u_{j}$. It is easy to see

$$
\begin{aligned}
& \sum_{j=1}^{n} k_{j} \leq m-|\gamma| \Leftrightarrow \sum_{j=1}^{n} k_{j} \leq m-\lceil|\gamma|\rceil ; \\
& \sum_{j=1}^{n} l_{j}<m-|\gamma| \Leftrightarrow \sum_{j=1}^{n} l_{j} \leq m-\lfloor|\gamma|\rfloor-1 .
\end{aligned}
$$

Again, the numbers of non-negative integer solutions of the above inequalities are the binomial coefficients $\left(\begin{array}{c}m-\lceil|\gamma| 7 \\ n\end{array}\right)$ and $\left(\begin{array}{c}m-\lfloor|\gamma|\rfloor-1 \\ n\end{array}\right)$, respectively; $D(\sigma, \Lambda)$ and $\bar{D}(\sigma, \Lambda)$ are in one-to-one correspondence, sending $u=\left(u_{1}, \ldots, u_{n}\right)$ to $\left(1-u_{1}, \ldots, 1-u_{n}\right)$. We thus have the following formulas on counting lattice points.

Theorem 2.5 [9]. Let $\sigma$ be an $n$-simplex whose vertices are points of $\Lambda$. Then

$$
\begin{aligned}
L(\sigma, \Lambda, m) & =\sum_{\gamma \in D\left(\sigma, \alpha_{0} ; \Lambda\right)}\left(\begin{array}{c}
m+n-\lceil|\gamma|\rceil \\
n
\end{array}\right) ; \\
L\left(\sigma^{0}, \Lambda, m\right) & =\sum_{\gamma \in \bar{D}\left(\sigma, \alpha_{0} ; \Lambda\right)}\left(\begin{array}{c}
m+n-1-\lfloor|\gamma|\rfloor \\
n
\end{array}\right) \\
& =\sum_{\gamma \in D\left(\sigma, \alpha_{0} ; \Lambda\right)}\left(\begin{array}{c}
m+\lceil|\gamma|\rceil-1 \\
n
\end{array}\right) .
\end{aligned}
$$

Moreover, the $j$ th coefficients of $L\left(\sigma^{0}, m\right)$ and $L(\sigma, m)$ are respectively given by

$$
\begin{aligned}
c_{j}\left(\sigma^{0}, \Lambda\right) & =\frac{1}{n !} \sum_{\gamma \in D\left(\sigma, \alpha_{0} ; \Lambda\right)} s_{n-j}(\lceil|\gamma|\rceil-1,\lceil|\gamma|\rceil-2, \ldots,\lceil|\gamma|\rceil-n) ; \\
c_{j}(\sigma, \Lambda) & =\frac{1}{n !} \sum_{\gamma \in D\left(\sigma, \alpha_{0} ; \Lambda\right)} s_{n-j}(1-\lceil|\gamma|\rceil, 2-\lceil|\gamma|\rceil, \ldots, n-\lceil|\gamma|\rceil) .
\end{aligned}
$$

Counting lattice points is also invariant under unimodular transformations. Let $A$ be an $N \times n$ matrix whose columns are the vectors $\alpha_{1}-\alpha_{0}, \ldots, \alpha_{n}-\alpha_{0}$. The matrix $A$ can be transformed into an upper triangular matrix by multiplying an $N \times N$ unimodular matrix to the left. This can be done by using repeatedly the modified Gaussian elimination and the Euclidean algorithm. Interchanging columns of $A$ is just to interchange the order of vertices of the simplex $\sigma$ with $\alpha_{0}$ fixed; we can also switch the columns of $A$ and have the number of lattice points inside $\sigma$ unchanged. Reversing the columns and the first $n$ rows of the upper triangular matrix (7), one can obtain a lower triangular form which is considered in [8]; we do not choose this lower triangular form in our treatment.

Theorem 2.6. Let the $N \times n$ matrix $A$ be in the upper triangular form (7) and let $u=\left(u_{1}, \ldots, u_{n}\right) \in[0,1)^{n}$. Then $A u \in \mathbf{Z}^{N}$ if and only if there exists $\left(j_{1}, \ldots, j_{n}\right) \in$ 
$\prod_{i=1}^{n}\left[0, a_{i i}\right) \cap \mathbf{Z}$ such that

$$
\left\{\begin{aligned}
u_{n}= & \frac{j_{n}}{u_{n n}}, \\
u_{i}= & \frac{\left\lceil a_{i(i+1)} u_{i+1}+\cdots+a_{i n} u_{n}\right\rceil}{a_{i i}} \\
& -\frac{a_{i(i+1)} u_{i+1}+\cdots+a_{i n} u_{n}}{a_{i i}}+\frac{j_{i}}{a_{i i}}, \quad 1 \leq i \leq n-1 .
\end{aligned}\right.
$$

In particular, $A u \in \mathbf{Z}^{N}$ implies that $u_{i}$ must be certain rational numbers of the form $j /\left(a_{i i} \cdots a_{n n}\right)$. Moreover, the map $\tilde{u}:\left(j_{1}, \ldots, j_{n}\right) \mapsto \sum_{i=1}^{n} u_{i}\left(\alpha_{i}-\alpha_{0}\right)$, where $u_{i}$ are given by (31), is a one-to-one correspondence between $\prod_{i=1}^{n}\left[0, a_{i i}\right) \cap \mathbf{Z}$ and $D\left(\sigma, \alpha_{0}\right)$.

Proof. Obviously, $u_{n}$ can have values $j_{n} / a_{n n}, 0 \leq j_{n} \leq a_{n n}-1$. Assume $u_{i+1}, \ldots, u_{n}$ are given values, we determine the possible values for $u_{i}$. Let $J_{i}$ be the smallest integer such that

$$
a_{i i} u_{i}^{\prime}+a_{i(i+1)} u_{i+1}+\cdots+a_{i n} u_{n}=J_{i}
$$

for some real number $u_{i}^{\prime} \in[0,1)$. Then

$$
\frac{J_{i}}{a_{i i}}-\frac{a_{i(i+1)} u_{i+1}+\cdots+a_{i n} u_{n}}{a_{i i}} \geq 0
$$

and

$$
\frac{J_{i}-1}{a_{i i}}-\frac{a_{i(i+1)} u_{i+1}+\cdots+a_{i n} u_{n}}{a_{i i}}<0 .
$$

This implies that $J_{i}=\left\lceil a_{i(i+1)} u_{i+1}+\cdots+a_{i n} u_{n}\right\rceil$. Thus $u_{i}$ can take the values

$$
\frac{\left\lceil a_{i(i+1)} u_{i+1}+\cdots+a_{i n} u_{n}\right\rceil}{a_{i i}}-\frac{a_{i(i+1)} u_{i+1}+\cdots+a_{i n} u_{n}}{a_{i i}}+\frac{j_{i}}{a_{i i}},
$$

where $0 \leq j_{i} \leq a_{i i}-1$, because when $j_{i}=a_{i i}-1$,

$$
\frac{\left\lceil a_{i(i+1)} u_{i+1}+\cdots+a_{i n} u_{n}\right\rceil}{a_{i i}}-\frac{a_{i(i+1)} u_{i+1}+\cdots+a_{i n} u_{n}}{a_{i i}}+\frac{a_{i i}-1}{a_{i i}}<1,
$$

and when $j_{i}=a_{i i}$,

$$
\frac{\left\lceil a_{i(i+1)} u_{i+1}+\cdots+a_{i n} u_{n}\right\rceil}{a_{i i}}-\frac{a_{i(i+1)} u_{i+1}+\cdots+a_{i n} u_{n}}{a_{i i}}+\frac{a_{i i}}{a_{i i}} \geq 1 .
$$

By definition of $D\left(\sigma, \alpha_{0}\right)$, the map $\tilde{u}$ is a one-to-one correspondence.

Now in Theorem 2.6, if the matrix $A$ is viewed as a group homomorphism from $\mathbf{Z}^{n}$ to $\mathbf{Z}^{N}$, the product $a_{11} \cdots a_{n n}$ of non-zero diagonal entries is the order of the torsion subgroup of $\mathbf{Z}^{N} / \operatorname{Im} A$. The transpose $A^{\mathrm{T}}$ is a group homomorphism from $\mathbf{Z}^{N}$ to $\mathbf{Z}^{n}$; and $\left|\mathbf{Z}^{n} / \operatorname{Im} A^{\mathrm{T}}\right|=a_{11} \cdots a_{n n}$. We define torsion for any non-zero integral matrix in the following. 
Definition 2.7. Let $\varphi$ be a group homomorphism from $\mathbf{Z}^{n}$ to $\mathbf{Z}^{m}$. The torsion of $\varphi$ is the order of the torsion subgroup of the quotient Abelian group $\mathbf{Z}^{m} / \operatorname{Im} \varphi$. If $A$ is the matrix of $\varphi$ under the standard bases, the torsion of $A$ is defined to be the torsion of $\varphi$, denoted tor $A=\operatorname{tor} \varphi$.

The torsion of a group homomorphism $\varphi: \mathbf{Z}^{n} \rightarrow \mathbf{Z}^{m}$ can be computed from its matrix under the standard bases. Notice that changing the bases of $\mathbf{Z}^{n}$ and $\mathbf{Z}^{m}$ corresponds to the modified Gaussian elimination: (a) adding an integral multiple of one row (column) to another row (column); (b) switching two rows (columns). By repeated use of the Euclidean algorithm and the modified Gaussian elimination, any integral matrix can be reduced to a Hermite normal form whose non-zero entries are all on the diagonal line, see [17]; the product of these non-zero entries is just the torsion of $\varphi$. Theorem 2.6 immediately gives a rough upper bound on the number of lattice points of a lattice simplex $\sigma$.

Corollary 2.8. Let $\sigma$ be a lattice $n$-simplex of $\mathbf{R}^{N}$ with vertices $\alpha_{0}, \alpha_{1}, \ldots, \alpha_{n}$; and let $A$ be the $N \times n$ matrix whose columns are vectors $\alpha_{1}-\alpha_{0}, \ldots, \alpha_{n}-\alpha_{0}$. Then $\left|D\left(\sigma, \alpha_{0}\right)\right|=$ tor $A$ and $\left|\sigma \cap \mathbf{Z}^{N}\right| \leq n+$ tor $A$.

\section{The Generating Functions of $L(\sigma, m)$ and $L\left(\sigma^{0}, m\right)$}

Let $\Lambda$ be a full-dimensional lattice of $\mathbf{R}^{N}$ with a lattice basis $\left\{\xi_{1}, \ldots, \xi_{N}\right\}$. Let $x=$ $\left(x_{1}, \ldots, x_{N}\right)$ be an undetermined multi-variable. For any $\alpha=a_{1} \xi_{1}+\cdots+a_{N} \xi_{N} \in \Lambda$, define the Laurent monomial $x^{\alpha}=x_{1}^{a_{1}} \cdots x_{N}^{a_{N}}$; for a polyhedron $P$ with vertices in $\Lambda$, define the formal power series

$$
F(P, \Lambda, x)=\sum_{\alpha \in P \cap \Lambda} x^{\alpha},
$$

which is an element of $\mathbf{C}\left[\left[x_{1}, \ldots, x_{N}, x_{1}^{-1}, \ldots, x_{N}^{-1}\right]\right]$. As usual we identify rational functions of $x$ as elements of $\mathbf{C}\left[\left[x_{1}, \ldots, x_{N}, x_{1}^{-1}, \ldots, x_{N}^{-1}\right]\right]$.

Let $K$ be a convex cone generated by vectors $\alpha_{1}, \ldots, \alpha_{n}$ of $\Lambda$, i.e., $K=\bigoplus_{j=1}^{n} \mathbf{R}_{\geq 0} \alpha_{j}$. Let $K^{0}=K-\partial K$ be the relative interior of $K$. Define the determined sets of $K$ by

$$
\begin{gathered}
D(K, \Lambda)=\left\{\sum_{j=1}^{n} u_{j} \alpha_{j} \in \Lambda: 0 \leq u_{j}<1\right\} ; \\
\bar{D}\left(K^{0}, \Lambda\right)=\left\{\sum_{j=1}^{n} v_{j} \alpha_{j} \in \Lambda: 0<v_{j} \leq 1\right\} .
\end{gathered}
$$

It is easy to see that a point $\alpha$ of $K$ (or $K^{0}$ ) belongs to $\Lambda$ if and only if there exist a unique vector $\gamma$ of $D(K, \Lambda)$ (or $\bar{D}(K, \Lambda)$ ) and non-negative integers $k_{j}$ such that $\alpha=\gamma+\sum_{j=1}^{n} k_{j} \alpha_{j}$. Then

$$
\begin{aligned}
F(K, \Lambda, x) & =\sum_{\gamma \in D(K, \Lambda)} \sum_{k_{1} \geq 0, \ldots, k_{n} \geq 0} x^{\gamma+k_{1} \alpha_{1}+\cdots+k_{n} \alpha_{n}} \\
& =\left(\sum_{\gamma \in D(K, \Lambda)} x^{\gamma}\right) \prod_{j=1}^{n} \frac{1}{1-x^{\alpha_{j}}} ;
\end{aligned}
$$




$$
\begin{aligned}
L\left(K^{0}, \Lambda, x\right) & =\left(\sum_{\gamma \in \bar{D}(K, \Lambda)} x^{\gamma}\right) \prod_{j=1}^{n} \frac{1}{1-x^{\alpha_{j}}} \\
& =\left(\sum_{\gamma \in D(K, \Lambda)} x^{-\gamma}\right) x^{\alpha_{1}+\cdots+\alpha_{n}} \prod_{j=1}^{n} \frac{1}{1-x^{\alpha_{j}}} .
\end{aligned}
$$

Throughout this section we denote by $\sigma$ an $n$-simplex of $\mathbf{R}^{N}$ with the vertex set $\left\{\alpha_{j}\right\}_{j=0}^{n} \subset \Lambda$. We put $\left\{\alpha_{j}\right\}_{j=0}^{n}$ in $\mathbf{R}^{N+1}$ in the hyperplane $x_{0}=1$ by lifting upward one unit to have independent vectors $\left\{\beta_{j}=\left(1, \alpha_{j}\right)\right\}_{j=0}^{n}$. Let $\sigma_{1}$ denote the $n$-simplex with the vertex set $\left\{\beta_{j}\right\}_{j=0}^{n}$. Let $\Lambda_{1}$ be the lattice generated by $\Lambda$ and the vector $\xi_{0}=$ $(1,0, \ldots, 0) \in \mathbf{R}^{N+1}$; then $\left\{\xi_{0}, \xi_{1}, \ldots, \xi_{N}\right\}$ is a lattice basis of $\Lambda_{1}$; and the number of points in $m \sigma \cap \Lambda$ is the same as the number of points in $m \sigma_{1} \cap \Lambda_{1}$. Set $V=\bigoplus_{j=0}^{n} \mathbf{R} \beta_{j}, M=V \cap \Lambda_{1}, C=\bigoplus_{j=0}^{n} \mathbf{R}_{\geq 0} \beta_{j}$; the lattice cone $C \cap \Lambda_{1}$ is a disjoint union of $m \sigma_{1} \cap \Lambda_{1}, m \geq 0$. Set $x_{0}=t$ and $x_{1}=\cdots=x_{N}=1$ in (32) and (34); we have the following generating functions for the sequences $L(\sigma, \Lambda, m)$ and $L\left(\sigma^{0}, \Lambda, m\right)$.

Proposition 3.1. Let $D\left(\sigma_{1}, \Lambda_{1}, k\right)$ be the determined set of the simplex $\sigma_{1}$ at level $k$ as defined in (17). Then

$$
\begin{aligned}
\sum_{m=0}^{\infty} L(\sigma, \Lambda, m) t^{m} & =\frac{1}{(1-t)^{n+1}} \sum_{\gamma \in D\left(C, \Lambda_{1}\right)} t^{|\gamma|} \\
& =\frac{1}{(1-t)^{n+1}} \sum_{k=0}^{n}\left|D\left(\sigma_{1}, \Lambda_{1}, k\right)\right| t^{k} \\
\sum_{m=1}^{\infty} L\left(\sigma^{0}, \Lambda, m\right) t^{m} & =\frac{1}{(1-t)^{n+1}} \sum_{\gamma \in \bar{D}\left(C, \Lambda_{1}\right)} t^{|\gamma|} \\
& =\frac{t^{n+1}}{(1-t)^{n+1}} \sum_{k=0}^{n}\left|D\left(\sigma_{1}, \Lambda_{1}, k\right)\right| t^{-k}
\end{aligned}
$$

Formulas (32)-(36) can be found in [20]. Let $A$ be the $N \times n$ matrix whose columns are the vectors $\left\{\alpha_{j}-\alpha_{0}\right\}_{j=0}^{n}$; and let $B$ be the $(N+1) \times(n+1)$ matrix whose columns are the vectors $\left\{\beta_{j}\right\}_{j=0}^{n}$. If $A$ is in upper triangular form (7), then $B$ is also in upper triangular form. The lattice points of the determined set $D\left(C, \Lambda_{1}\right)$ can be listed by a one-to-one correspondence $\tilde{u}: \prod_{i=0}^{n}\left[0, a_{i i}\right) \cap \mathbf{Z} \rightarrow D\left(C, \Lambda_{1}\right)$ similar to $\tilde{u}\left(j_{1}, \ldots, j_{n}\right)$. In fact, for any $\left(u_{0}, u_{1}, \ldots, u_{n}\right) \in[0,1)^{n+1}, \sum_{i=0}^{n} u_{i} \beta_{i} \in \mathbf{Z}^{N+1}$ if and only if both $\sum_{i=0}^{n} u_{i} \in \mathbf{Z}$ and $\sum_{i=1}^{n} u_{i} \alpha_{i} \in \mathbf{Z}^{N}$. Since $0 \leq u_{0}<1$, we have $u_{0}=\left\lceil\sum_{i=1}^{n} u_{i}\right\rceil-\sum_{i=1}^{n} u_{i}$. Thus $\tilde{u}\left(j_{0}, j_{1}, \ldots, j_{n}\right)=\sum_{i=0}^{n} u_{i}\left(\alpha_{i}-\alpha_{0}\right)$, where $\left(u_{i}\right)_{i=1}^{n}$ are given by (8) or (31), $u_{0}=\left\lceil\sum_{i=1}^{n} u_{i}\right\rceil-\sum_{i=1}^{n} u_{i}, j_{0} \equiv 1$. We have the following corollary. 
Corollary 3.2. Let $A$ be the matrix whose columns are the vectors $\alpha_{1}-\alpha_{0}, \ldots, \alpha_{n}-\alpha_{0}$. If $A$ is in upper triangular form (7), then

$$
\begin{aligned}
\sum_{m=0}^{\infty} L(\sigma, \Lambda, m) t^{m} & =\frac{1}{(1-t)^{n+1}} \sum_{j_{1}=0}^{a_{11}-1} \cdots \sum_{j_{n}=0}^{a_{n n}-1} t^{\left[\left|u\left(j_{1}, \ldots, j_{n}\right)\right|\right\rceil}, \\
\sum_{m=1}^{\infty} L(\sigma, \Lambda, m) t^{m} & =\frac{t^{n+1}}{(1-t)^{n+1}} \sum_{j_{1}=0}^{a_{11}-1} \cdots \sum_{j_{n}=0}^{a_{n n}-1} t^{-\left[\left|u\left(j_{1}, \ldots, j_{n}\right)\right|\right\rceil},
\end{aligned}
$$

where $u\left(j_{1}, \ldots, j_{n}\right)=\left(u_{1}, \ldots, u_{n}\right)$ is defined by $(8)$.

If we do not use the one-to-one correspondence $\tilde{u}$ by (8) to list all lattice points of the determined set $D\left(C, \Lambda_{1}\right)$, one may refine $D\left(C, \Lambda_{1}\right)$ so that all lattice points inside can be listed canonically at the cost of using characters. To this end, we need the following lemma.

Lemma 3.3. Let $L_{1}$ and $M_{1}$ be lattices of finite rank of a vector space $V_{1}$; let $L_{2}$ and $M_{2}$ be lattices of the same rank of a vector space $V_{2}$ such that $L_{2} \subset M_{2} ; G_{2}=M_{2} / L_{2}$. Let $\langle$,$\rangle be a bilinear pairing V_{1} \times V_{2} \rightarrow \mathbf{R}$ such that $M_{1} \times M_{2} \rightarrow \mathbf{Z}$.

(i) If $L_{1} \times L_{2} \rightarrow \mathbf{Z}$, then the character map $\chi: L_{1} \times G_{2} \rightarrow \mathbf{C}^{*}$ by $\chi_{g}(\alpha)=e^{2 \pi \imath\langle\alpha, g\rangle}$ is a well-defined group bi-homomorphism.

(ii) For $\alpha \in L_{1}-M_{1}$, if there exists $g_{\alpha} \in M_{2}$ such that $\left\langle\alpha, g_{\alpha}\right\rangle \notin \mathbf{Z}$, then

$$
\sum_{g \in G} \chi_{g}(\alpha)=0
$$

(iii) Let $\left\{\gamma_{j}\right\}_{j=0}^{n}$ be a lattice basis of $L_{1} ; C=\bigoplus_{j=0}^{n} \mathbf{R}_{\geq 0} \gamma_{j}, C^{0}=\bigoplus_{j=0}^{n} \mathbf{R}_{>0} \gamma_{j}$. If $L_{1} \times L_{2} \rightarrow \mathbf{Z}, C \cap M_{1} \subset C \cap L_{1}$, and for each $\alpha \in C \cap\left(L_{1}-M_{1}\right)$ there exists $g_{\alpha} \in M_{2}$ such that $\left\langle\alpha, g_{\alpha}\right\rangle \notin \mathbf{Z}$, then

$$
\begin{gathered}
F\left(C, M_{1}, x\right)=\sum_{\alpha \in C \cap M_{1}} x^{\alpha}=\frac{1}{\left|G_{2}\right|} \sum_{g \in G_{2}} \prod_{j=0}^{n} \frac{1}{1-\chi_{g}\left(\gamma_{j}\right) x^{\gamma_{j}}} ; \\
F\left(C^{0}, M_{1}, x\right)=\sum_{\alpha \in C^{0} \cap M_{1}} x^{\alpha}=\frac{1}{\left|G_{2}\right|} \sum_{g \in G_{2}} \prod_{j=0}^{n} \frac{\chi_{g}\left(\gamma_{j}\right) x^{\gamma_{j}}}{1-\chi_{g}\left(\gamma_{j}\right) x^{\gamma_{j}}} .
\end{gathered}
$$

Proof. (i) When we write $\langle\alpha, g\rangle$ for $\alpha \in L_{1}$ and $g \in G_{2}$, we understand that $g$ is taken to be a representative of its coset $g+L_{2}$. Now it is trivial that $\chi_{g}(\alpha)$ is a bi-homomorphism.

(ii) Let $\alpha$ be a vector of $L_{1}-M_{1}$ such that $\langle\alpha, g\rangle \notin \mathbf{Z}$ for some $g \in M_{2}$. Denote by $d(g)$ the order of $g$ in $G_{2}$. Since $\chi_{g}(\alpha)=e^{2 \pi \imath\langle\alpha, g\rangle}$ is a root of unity, then $\sum_{j=1}^{d(g)} \chi_{j g}(\alpha)=0$. Fix one such group element $g^{\prime}$ and let $\left\langle g^{\prime}\right\rangle$ denote the cyclic group generated by $g^{\prime}$; write $G_{2}$ as a disjoint union $G_{2}=\bigcup_{k}\left(g_{k}+\left\langle g^{\prime}\right\rangle\right)$ of cosets for some representatives $g_{k}$. Obviously,

$$
\sum_{g \in G_{2}} \chi_{g}(\alpha)=\sum_{k} \chi_{g_{k}}(\alpha) \sum_{g \in\left\langle g^{\prime}\right\rangle} \chi_{g}(\alpha)=0
$$


(iii) Let $\alpha \in C \cap L_{1}$. If $\alpha \in M_{1}$, then $\chi_{g}(\alpha)=1$ for all $g \in G_{2}$. It follows from (ii) that

$$
\frac{1}{\left|G_{2}\right|} \sum_{g \in G_{2}} \chi_{g}(\alpha)=\left\{\begin{array}{lll}
1 & \text { for } & \alpha \in M_{1} \\
0 & \text { for } & \alpha \notin M_{1}
\end{array}\right.
$$

Write $\alpha$ uniquely as $\alpha=\sum_{j=0}^{n} k_{j} \gamma_{j}$ for some $k_{j} \geq 0$; we have

$$
\begin{aligned}
F\left(C, M_{1}, x\right) & =\sum_{\alpha \in C \cap L_{1}} x^{\alpha} \frac{1}{\left|G_{2}\right|} \sum_{g \in G_{2}} \chi_{g}(\alpha) \\
& =\frac{1}{\left|G_{2}\right|} \sum_{g \in G_{2}} \sum_{\alpha \in C \cap L_{1}} \chi_{g}(\alpha) x^{\alpha} \\
& =\frac{1}{\left|G_{2}\right|} \sum_{g \in G_{2}} \sum_{k_{1}=0}^{\infty} \cdots \sum_{k_{n}=0}^{\infty} \chi_{g}\left(\sum_{j=0}^{n} k_{j} \gamma_{j}\right) x^{\sum_{j=0}^{n} k_{j} \gamma_{j}} \\
& =\frac{1}{\left|G_{2}\right|} \sum_{g \in G_{2}} \sum_{k_{1}=0}^{\infty} \cdots \sum_{k_{n}=0}^{\infty} \prod_{j=0}^{n} \chi_{g}^{k_{j}}\left(\gamma_{j}\right)\left(x^{\gamma_{j}}\right)^{k_{j}} \\
& =\frac{1}{\left|G_{2}\right|} \sum_{g \in G_{2}} \prod_{j=0}^{n} \frac{1}{1-\chi_{g}\left(\gamma_{j}\right) x^{\gamma_{j}}} .
\end{aligned}
$$

The situation for $F\left(C^{0}, M_{1}, x\right)$ is similar, just pay attention to $k_{0} \geq 1, k_{1} \geq 1, \ldots, k_{n+1}$ $\geq 1$ in the above summation.

Theorem 3.4. Let $V=\bigoplus_{j=0}^{n} \mathbf{R} \beta_{j}, M=V \cap \Lambda_{1}, C=\bigoplus_{j=0}^{n} \mathbf{R}_{\geq 0} \beta_{j}$; and let $p_{j}$ be positive integers such that $C \cap M \subset C \cap L$, where $L=\bigoplus_{j=0}^{n} \mathbf{Z} \beta_{j} / p_{j}$. Let $L^{\prime}$ and $M^{\prime}$ be lattices of the same rank of a vector space $V^{\prime}$ such that $L^{\prime} \subset M^{\prime} ; G=M^{\prime} / L^{\prime}$. Let $\langle$,$\rangle be a bilinear pairing V \times V^{\prime} \rightarrow \mathbf{R}$ such that $M \times M^{\prime} \rightarrow \mathbf{Z}$ and $L \times L^{\prime} \rightarrow \mathbf{Z}$. If for any $\alpha \in C \cap(L-M)$ there exists $g_{\alpha} \in M^{\prime}$ such that $\left\langle\alpha, g_{\alpha}\right\rangle \notin \mathbf{Z}$, then

$$
\begin{aligned}
\sum_{m=0}^{\infty} L(\sigma, \Lambda, m) e^{2 \pi m s} & =\frac{1}{2^{n+1}|G|} \sum_{g \in G} \prod_{j=0}^{n}\left(1+\operatorname{coth} \frac{\pi}{p_{j}}\left(s+\imath\left\langle\beta_{j}, g\right\rangle\right)\right) \\
\sum_{m=1}^{\infty} L\left(\sigma^{0}, \Lambda, m\right) e^{2 \pi m s} & =\frac{(-1)^{n+1}}{2^{n+1}|G|} \sum_{g \in G} \prod_{j=0}^{n}\left(1-\operatorname{coth} \frac{\pi}{p_{j}}\left(s+\imath\left\langle\beta_{j}, g\right\rangle\right)\right) .
\end{aligned}
$$

Proof. This is an immediate consequence of Lemma 3.3. Set $V_{1}=V, M_{1}=M$, $L_{1}=L, V_{2}=V^{\prime}, M_{2}=M^{\prime}, L_{2}=L^{\prime}, x=\left(e^{-2 \pi s}, 1, \ldots, 1\right), \gamma_{j}=\beta_{j} / p_{j}$; apply (iii) of Lemma 3.3, and keep in mind

$$
\frac{1}{1-e^{-2 z}}=\frac{1}{2}(1+\operatorname{coth} z), \quad \frac{e^{-2 z}}{1-e^{-2 z}}=-\frac{1}{2}(1-\operatorname{coth} z) .
$$


We then have

$$
\begin{aligned}
\frac{1}{1-\chi_{g}\left(\gamma_{j}\right) x^{\gamma_{j}}} & =\frac{1}{1-\exp \left(-\left(2 \pi / p_{j}\right)\left(s+\imath\left\langle\beta_{j}, g\right\rangle\right)\right)} \\
& =\frac{1}{2}\left(1+\operatorname{coth} \frac{\pi}{p_{j}}\left(s+\imath\left\langle\beta_{j}, g\right\rangle\right)\right) ; \\
\frac{\chi_{g}\left(\gamma_{j}\right) x^{\gamma_{j}}}{1-\chi_{g}\left(\gamma_{j}\right) x^{\gamma_{j}}} & =\frac{\exp \left(-\left(2 \pi / p_{j}\right)\left(s+\imath\left\langle\beta_{j}, g\right\rangle\right)\right)}{1-\exp \left(-\left(2 \pi / p_{j}\right)\left(s+\imath\left\langle\beta_{j}, g\right\rangle\right)\right)} \\
& =-\frac{1}{2}\left(1-\operatorname{coth} \frac{\pi}{p_{j}}\left(s+\imath\left\langle\beta_{j}, g\right\rangle\right)\right) .
\end{aligned}
$$

Formulas (41) and (42) are naively simple, but they are very useful. A notable feature is that the group $G$ can be selected as long as the lattices $L, L^{\prime}, M, M^{\prime}$ satisfy the required conditions. One can fix a pairing $V \times V^{\prime} \rightarrow \mathbf{R}$ satisfying $M \times M^{\prime} \rightarrow \mathbf{Z}$, then construct a lattice $L$ such that $C \cap M \subset C \cap L$. Since $L$ may contain some points not in $M$, usually we do not have $L \times M^{\prime} \rightarrow \mathbf{Z}$. However, there always exists a sublattice $L^{\prime} \subset M^{\prime}$ of the same rank such that $L \times L^{\prime} \rightarrow \mathbf{Z}$; and any sublattice $L^{\prime \prime} \subset L^{\prime}$ of the same rank does the same job. We give several examples for Theorem 3.4 in the following.

Example 3.5 [8]. Let $\sigma$ be a lattice $n$-simplex of $\mathbf{R}^{n}$ with the vertex set $\left\{\alpha_{j}\right\}_{j=0}^{n} \subset \mathbf{Z}^{n}$. Let $A$ be the matrix whose columns are the vectors $\left\{\alpha_{j}-\alpha_{0}\right\}_{j=0}^{n}$; and let $B$ be the matrix whose columns are the vectors $\left\{\beta_{j}=\left(1, \alpha_{j}\right)\right\}_{j=0}^{n}$. Let $A$ be in upper triangular form (7); set $p_{j}=a_{j j} \cdots a_{n n}, 0 \leq j \leq n$, where $a_{00}=1 ; G=\prod_{j=0}^{n} \mathbf{Z} / p_{j} \mathbf{Z}$. Then we have (41) and (42).

Proof. Set $N=n, V=V^{\prime}=\mathbf{R}^{n+1}, M=M^{\prime}=\Lambda_{1}=\mathbf{Z}^{n+1}$. The pairing $V \times V^{\prime} \rightarrow \mathbf{R}$ is given by the inner product $\langle$,$\rangle of \mathbf{R}^{n+1}$, which is defined by $\langle\alpha, \beta\rangle=\sum_{j=0}^{n} u_{j} v_{j}$ for $\alpha=\sum_{j=0}^{n} u_{j} e_{j}$ and $\beta=\sum_{j=0}^{n} v_{j} e_{j}$, where $\left\{e_{j}\right\}_{j=0}^{n}$ is the standard lattice basis of $\mathbf{Z}^{n+1}$. Obviously, $M \times M^{\prime} \rightarrow \mathbf{Z}$. Set $\gamma_{j}=\beta_{j} / p_{j}, \gamma_{j}^{\prime}=p_{j} e_{j}, L=\sum_{j=0}^{n} \mathbf{Z} \gamma_{j}, L^{\prime}=\sum_{k=0}^{n} \mathbf{Z} \gamma_{k}^{\prime}$. By Theorem 2.6, $C \cap M \subset C \cap L$. Since

$$
\left\langle\gamma_{j}, \gamma_{k}^{\prime}\right\rangle= \begin{cases}\frac{a_{k j} a_{k k} \cdots a_{n n}}{a_{j j} \cdots a_{n n}} & \text { for } j \geq k, \\ 0 & \text { for } j<k\end{cases}
$$

are integers, we have $L \times L^{\prime} \rightarrow \mathbf{Z}$. For $\alpha \in C \cap(L-M)$, write $\alpha=\sum_{j=0}^{n} u_{j} e_{j}$; since $\alpha \notin M$, some $u_{j}$ must be non-integral, say $u_{j^{\prime}}$; we have $e_{j^{\prime}} \in M^{\prime}$ and $\left\langle\alpha, e_{j^{\prime}}\right\rangle=u_{j^{\prime}} \notin \mathbf{Z}$. Finally, $G=M^{\prime} / L^{\prime}=\mathbf{Z}^{n+1} / \prod_{j=0}^{n} p_{j} \mathbf{Z}=\prod_{j=0}^{n} \mathbf{Z} / p_{j} \mathbf{Z}$. Now all the conditions of Theorem 3.4 are satisfied. We thus recover the main result of [8], which was first obtained by the Poisson summation and Fourier analysis.

Sometimes it may not be easy to check the conditions of Theorem 3.4. In particular, it is important to find a canonical way to check the conditions: $C \cap M \subset C \cap L$; the existence of $g_{\alpha} \in M^{\prime}$ such that $\left\langle\alpha, g_{\alpha}\right\rangle \notin \mathbf{Z}$ for each $\alpha \in C \cap(L-M)$; and $L_{1} \times L_{2} \rightarrow \mathbf{Z}$. The following lemma may help to do the job. 
Lemma 3.6. Let $M_{1}$ be a lattice with a lattice basis $\left\{\eta_{k}\right\}_{k=0}^{l}$ of a vector space $V_{1}$; and let $M_{2}$ be a lattice with a lattice basis $\left\{\eta_{k}^{\prime}\right\}_{k=0}^{l}$ of a vector space $V_{2}$. Let $\langle$,$\rangle be a bilinear$ pairing $V_{1} \times V_{2} \rightarrow \mathbf{R}$ such that $\left\langle\eta_{j}, \eta_{k}^{\prime}\right\rangle=\delta_{j k}$. Given independent vectors $\left\{\beta_{j}\right\}_{j=0}^{n}$ of $M_{1}$ and independent vectors $\left\{\beta_{j}^{\prime}\right\}_{j=0}^{n}$ of $M_{2}$ such that $\left\langle\beta_{j}, \beta_{k}^{\prime}\right\rangle=p_{j} \delta_{j k}$, where $p_{j}$ are positive integers. Set $C=\bigoplus_{j=0}^{n} \mathbf{R}_{\geq 0} \beta_{j}, \gamma_{j}=\beta_{j} / p_{j}, L_{1}=\bigoplus_{j=0}^{n} \mathbf{Z}_{\gamma_{j}}$. We have:

(i) $C \cap M_{1} \subset C \cap L_{1}$.

(ii) For any $\alpha \in C \cap\left(L_{1}-M_{1}\right)$, there exists $\varphi \in M_{2}$ such that $\langle\alpha, \varphi\rangle \notin \mathbf{Z}$.

(iii) Set $p=\operatorname{lcm}\left\{p_{j}\right\}_{j=0}^{n}, b_{k}=\operatorname{gcd}\left\{\left\langle\beta_{j}, \eta_{k}^{\prime}\right\rangle\right\}_{j=0}^{n}, q_{k}=p / \operatorname{gcd}\left(p, b_{k}\right), \gamma_{k}^{\prime}=q_{k} \eta_{k}^{\prime}$, $L_{2}=\bigoplus_{k=0}^{l} \mathbf{Z} \gamma_{k}^{\prime}$. Then $L_{1} \times L_{2} \rightarrow \mathbf{Z}$.

Proof. (i) For any $\beta \in C \cap M_{1}$, write $\beta=\sum_{j=0}^{n} v_{j} \gamma_{j}$. Since $\beta \in M_{1}$ and $\beta_{k}^{\prime} \in M_{2}$, $\left\langle\beta, \beta_{k}^{\prime}\right\rangle$ is an integer; but $\left\langle\beta, \beta_{k}^{\prime}\right\rangle=\left\langle\sum_{j=0}^{n} v_{j} \gamma_{j}, \beta_{k}^{\prime}\right\rangle=v_{k}$. This shows that $\beta \in L_{1}$, so $\beta \in C \cap L_{1}$.

(ii) Let $\alpha \in C \cap\left(L_{1}-M_{1}\right)$. Since $\alpha$ is a linear combination of $\left\{\beta_{j}\right\}_{j=0}^{n}$, it is a linear combination of $\left\{\eta_{k}\right\}_{k=0}^{l}$. Write $\alpha=\sum_{k=0}^{l} u_{k} \eta_{k}$; since $\alpha \notin M_{1}$, some $u_{k}$ must be non-integral, say $u_{k^{\prime}}$. We thus have $\eta_{k^{\prime}}^{\prime} \in M_{2}$ and $\left\langle\alpha, \eta_{k^{\prime}}^{\prime}\right\rangle=\left\langle\sum_{k=0}^{l} u_{k} \eta_{k}, \eta_{k^{\prime}}^{\prime}\right\rangle=u_{k^{\prime}} \notin \mathbf{Z}$.

(iii) The result follows from

$$
\left\langle\gamma_{j}, \gamma_{k}^{\prime}\right\rangle=\frac{\operatorname{lcm}\left\{p_{i}\right\}_{i=0}^{n}}{p_{j}} \cdot \frac{\left\langle\beta_{j}, \eta_{k}^{\prime}\right\rangle}{\operatorname{gcd}\left(p, b_{k}\right)} \in \mathbf{Z}, \quad 0 \leq j \leq n, \quad 0 \leq k \leq l .
$$

Example 3.7. Let $V=\bigoplus_{j=0}^{n} \mathbf{R} \beta_{j}, M=V \cap \Lambda_{1}, C=\bigoplus_{j=0}^{n} \mathbf{R}_{\geq 0} \beta_{j}$. Let $V^{*}$ be the dual vector space of $V$ and let $M^{*}$ be the dual lattice of $M$, i.e., $V^{*}$ is the vector space of all linear functionals on $V$ and

$$
M^{*}=\left\{\varphi \in V^{*}:\langle\alpha, \varphi\rangle=\varphi(\alpha) \in \mathbf{Z} \text { for all } \alpha \in M\right\} .
$$

Let $\beta_{j}^{\prime} \in M^{*}$ be a normal vector of the facet of $C$ without $\beta_{j}$ such that $\left\langle\beta_{j}, \beta_{j}^{\prime}\right\rangle=p_{j}$ is positive, i.e., $\left\langle\beta_{j}, \beta_{k}^{\prime}\right\rangle=p_{j} \delta_{j k}, p_{j}>0$. Set $L^{*}=\bigoplus_{j=0}^{n} \mathbf{Z} \beta_{j}^{\prime}, G=M^{*} / L^{*}$. Then we have (41) and (42).

Proof. Set $V_{1}=V, M_{1}=M, L_{1}=\bigoplus_{j=0}^{n} \mathbf{Z} \gamma_{j}$, where $\gamma_{j}=\beta_{j} / p_{j}$; and $V_{2}=V_{1}^{*}$, $M_{2}=M_{1}^{*}, L_{2}=L_{1}^{*}$. Since $\left\langle\gamma_{j}, \beta_{k}^{\prime}\right\rangle=\delta_{j k}$, we have $L_{2}=L^{*}$. We then have a natural pairing $V_{1} \times V_{2} \rightarrow \mathbf{R}$ such that $M_{1} \times M_{2} \rightarrow \mathbf{Z}$ and $L_{1} \times L_{2} \rightarrow \mathbf{Z}$. Keep in mind that $l=n$; choose a basis $\left\{\eta_{k}\right\}_{k=0}^{n}$ of $M$ and its dual basis $\left\{\eta_{k}^{\prime}:=\eta_{k}^{*}\right\}_{k=0}^{n}$ of $M^{*}$, i.e., $\left\langle\eta_{j}, \eta_{k}^{\prime}\right\rangle=\delta_{j k}$; we then obtain the conclusions of Lemma 3.6.

Now we set $L=L_{1}, V^{\prime}=V_{2}, M^{\prime}=M^{*}, L^{\prime}=L_{2}$. Then all conditions of Theorem 3.4 on $V, V^{\prime}, M, M^{\prime}, L, L^{\prime}$ are satisfied and $G=M^{\prime} / L^{\prime}=M^{*} / L^{*}$. We thus have (41) and (42).

It is not easy to list the elements of the group $G=M^{*} / L^{*}$. However, the group $M^{*} / L^{*}$ has fewer elements than other groups that will be given later. In the following we apply Theorem 3.4 to find some more convenient groups to express the generating functions in terms of hyperbolic cotangent functions. Recall the lattice basis $\left\{\xi_{0}, \xi_{1}, \ldots, \xi_{N}\right\}$ of the 
lattice $\Lambda_{1}$ in $\mathbf{R}^{N+1}$; there is a canonical inner product on $\mathbf{R}^{N+1}$ : for $\alpha=\sum_{k=0}^{N} u_{k} \xi_{k}$ and $\beta=\sum_{k=0}^{N} v_{k} \xi_{k}$, define

$$
\langle\alpha, \beta\rangle=\sum_{k=0}^{N} u_{k} v_{k}
$$

Example 3.8. Let $C=\bigoplus_{j=0}^{n} \mathbf{R}_{\geq 0} \beta_{j}$; and let $\beta_{j}^{\prime} \in \Lambda_{1}$ be a normal vector of the facet of $C$ without $\beta_{j}$ such that $\left\langle\beta_{j}, \beta_{j}^{\prime}\right\rangle=p_{j}$ is positive, i.e., $\left\langle\beta_{j}, \beta_{k}^{\prime}\right\rangle=p_{j} \delta_{j k}, p_{j}>0$. Set $p=\operatorname{lcm}\left\{p_{j}\right\}_{j=0}^{n}, b_{k}=\operatorname{gcd}\left\{\left\langle\beta_{j}, \xi_{k}\right\rangle\right\}_{j=0}^{n}, q_{k}=p / \operatorname{gcd}\left\{p, b_{k}\right\} ; G=\prod_{k=0}^{N} \mathbf{Z} / q_{k} \mathbf{Z}$. Then we have (41) and (42).

Proof. Note that $p_{j}$ are integers because both $\beta_{j}$ and $\beta_{j}^{\prime}$ are points of $\Lambda_{1}$. Set $V_{1}=$ $V_{2}=\mathbf{R}^{N+1}, M_{1}=M_{2}=\Lambda_{1}, L_{1}=\bigoplus_{j=0}^{n} \mathbf{Z} \gamma_{j}, L_{2}=\bigoplus_{k=0}^{N} \gamma_{k}^{\prime}$, where $\gamma_{j}=\beta_{j} / p_{j}$, $\gamma_{k}^{\prime}=q_{k} \xi_{k}$; the inner product (43) gives a natural pairing $V_{1} \times V_{2} \rightarrow \mathbf{R}$. Keep in mind that $l=N, \eta_{k}=\eta_{k}^{\prime}=\xi_{k}$; then all conditions of Lemma 3.6 on $V_{1}, V_{2}, M_{1}, M_{2}, L_{1}, L_{2}$ are satisfied.

Now we set $V=\bigoplus_{j=0}^{n} \mathbf{R} \beta_{j}, M=V \cap \Lambda_{1}, L=L_{1}, V^{\prime}=V_{2}, M^{\prime}=M_{2}, L^{\prime}=L_{2}$. Obviously, the conditions of Theorem 3.4 on $V, V^{\prime}, M, M^{\prime}, L, L^{\prime}$ are all satisfied; and $M^{\prime} / L^{\prime}$ can be identified to $G=\prod_{k=0}^{N} \mathbf{Z} / q_{k} \mathbf{Z}$. We thus have (41) and (42).

Example 3.9. Let $B$ be the $(N+1) \times(n+1)$ matrix whose columns are the coordinate vectors of $\beta_{0}, \beta_{1}, \ldots, \beta_{n}$, respectively, under the lattice basis $\left\{\xi_{k}\right\}_{k=0}^{N}$. Set

$$
\begin{aligned}
a_{j} & =\operatorname{gcd} \text { of the } j \text { th row of } \operatorname{adj}\left(B^{\mathrm{T}} B\right) B^{\mathrm{T}}, \\
b_{k} & =\operatorname{gcd} \text { of the } k \text { th row of } B \operatorname{diag}\left[a_{0}, a_{1}, \ldots, a_{n}\right], \\
p_{j} & =\frac{\left|\operatorname{det} B^{\mathrm{T}} B\right|}{a_{j}}, \\
q_{k} & =\frac{\left|\operatorname{det} B^{\mathrm{T}} B\right|}{\operatorname{gcd}\left(b_{k}, \operatorname{det} B^{\mathrm{T}} B\right)} ;
\end{aligned}
$$

and $G=\prod_{k=0}^{N} \mathbf{Z} / q_{k} \mathbf{Z}$. Then we have (41) and (42).

Proof. Write the integral matrix $B$ as

$$
B=\left(\begin{array}{cccc}
b_{00} & b_{01} & \cdots & b_{0 n} \\
b_{10} & b_{11} & \cdots & b_{1 n} \\
\vdots & \vdots & & \vdots \\
b_{N 0} & b_{N 1} & \cdots & b_{N n}
\end{array}\right)
$$

Let $B^{\mathrm{T}}$ denote the transpose of $B$. Since $\beta_{0}, \beta_{1}, \ldots, \beta_{n}$ are linearly independent, the $(n+1) \times(n+1)$ matrix $B^{\mathrm{T}} B$ is non-singular. From the elementary theory of linear algebra, we have

$$
\operatorname{adj}\left(B^{\mathrm{T}} B\right)\left(B^{\mathrm{T}} B\right)=\operatorname{adj}\left(B^{\mathrm{T}} B\right) B^{\mathrm{T}} B=\left(\operatorname{det} B^{\mathrm{T}} B\right) I_{n+1},
$$


where $I_{n+1}$ is the $(n+1) \times(n+1)$ identity matrix. Without loss of generality we assume that $\operatorname{det} B^{\mathrm{T}} B$ is positive.

Set $V_{1}=V_{2}=\mathbf{R}^{N+1}, M_{1}=M_{2}=\Lambda_{1}, L_{1}=\bigoplus_{j=0}^{n} \mathbf{Z} \gamma_{j}, L_{2}=\bigoplus_{k=0}^{N} \mathbf{Z} \gamma_{k}^{\prime}$, where $\gamma_{j}=\beta_{j} / p_{j}, \gamma_{k}^{\prime}=q_{k} \xi_{k}$; the inner product (43) gives a natural pairing $V_{1} \times V_{2} \rightarrow \mathbf{R}$. Let $\beta_{j}^{\prime}$ be the $j$ th row of $\operatorname{adj}\left(B^{\mathrm{T}} B\right) B^{\mathrm{T}}$ divided by $a_{j}$. Then (44) shows that $\left\langle\beta_{j}, \beta_{k}^{\prime}\right\rangle=p_{j} \delta_{j k}$. Since both $\beta_{j}$ and $\beta_{j}^{\prime}$ are points of $\Lambda_{1}$, it implies that $p_{j}$ are integers. Keep in mind that $l=N, \eta_{k}=\eta_{k}^{\prime}=\xi_{k}$; we then obtain conclusions (i) and (ii) of Lemma 3.6. Note that

$$
\left\langle\gamma_{j}, \gamma_{k}^{\prime}\right\rangle=\frac{\left|\operatorname{det} B^{\mathrm{T}} B\right|}{p_{j} \operatorname{gcd}\left(b_{k}, \operatorname{det} B^{\mathrm{T}} B\right)}\left\langle\beta_{j}, \xi_{k}\right\rangle=\frac{a_{j} b_{k j}}{\operatorname{gcd}\left(b_{k}, \operatorname{det} B^{\mathrm{T}} B\right)} \in \mathbf{Z} .
$$

Then $L_{1} \times L_{2} \rightarrow \mathbf{Z}$.

Now we set $V=\bigoplus_{j=0}^{n} \mathbf{R} \beta_{j}, M=V \cap \Lambda_{1}, L=L_{1}, V^{\prime}=V_{2}, M^{\prime}=M_{2}, L^{\prime}=L_{2}$. Obviously, all conditions of Theorem 3.4 on $V, V^{\prime}, M, M^{\prime}, L, L^{\prime}$ are satisfied; and $M^{\prime} / L^{\prime}$ can be identified to $G=\prod_{k=0}^{N} \mathbf{Z} / q_{k} \mathbf{Z}$. We thus have (41) and (42).

Example 3.10. Let $A$ be the $N \times n$ matrix whose columns are the coordinate vectors of $\alpha_{1}-\alpha_{0}, \ldots, \alpha_{n}-\alpha_{0}$, respectively, under the lattice basis $\left\{\xi_{k}\right\}_{k=1}^{N}$; and let $B$ be the $(N+1) \times(n+1)$ matrix whose columns are the coordinate vectors of $\beta_{0}, \beta_{1}, \ldots, \beta_{n}$ respectively under the lattice basis $\left\{\xi_{k}\right\}_{k=0}^{N}$. Set $p=\operatorname{tor} A$; then

$$
\begin{aligned}
\sum_{m=0}^{\infty} L(\sigma, \Lambda, m) e^{-2 \pi m s}= & \frac{1}{2^{n+1} p^{N+1}} \\
& \times \sum_{\substack{h_{0}, h_{1}, \ldots, h_{N}=0 \\
h=\left(h_{0}, h_{1}, \ldots, h_{N}\right)}}^{p-1} \prod_{j=0}^{n}\left(1+\operatorname{coth} \frac{\pi}{p}\left(s+\imath\left\langle\beta_{j}, h\right\rangle\right)\right) ; \\
\sum_{m=1}^{\infty} L\left(\sigma^{0}, \Lambda, m\right) e^{-2 \pi m s}= & \frac{(-1)^{(n+1)}}{2^{n+1} p^{N+1}} \\
& \times \sum_{\substack{h_{0}, h_{1}, \ldots, h_{N}=0 \\
h=\left(h_{0}, h_{1}, \ldots, h_{N}\right)}}^{p-1} \prod_{j=0}^{n}\left(1-\operatorname{coth} \frac{\pi}{p}\left(s+\imath\left\langle\beta_{j}, h\right\rangle\right)\right) .
\end{aligned}
$$

Proof. $\quad$ Set $V=\bigoplus_{j=0}^{n} \mathbf{R} \beta_{j}, V^{\prime}=\mathbf{R}^{N+1}, M=V \cap \Lambda_{1}, M^{\prime}=\Lambda_{1}, C=\bigoplus_{j=0}^{n} \mathbf{R}_{\geq 0} \beta_{j} ;$ the inner product (43) gives a pairing $V \times V^{\prime} \rightarrow \mathbf{R}$ such that $M \times M^{\prime} \rightarrow \mathbf{Z}$. Set $\gamma_{j}=\beta_{j} / p$, $\gamma_{k}^{\prime}=p \xi_{k}, L=\bigoplus_{j=0}^{n} \mathbf{Z} \gamma_{j}, L^{\prime}=\bigoplus_{k=0}^{N} \mathbf{Z} \gamma_{k}^{\prime}$. By Theorem 2.6, $C \cap M \subset C \cap L$. Obviously, $\left\langle\gamma_{j}, \gamma_{k}^{\prime}\right\rangle=\left\langle\beta_{j}, \xi_{k}\right\rangle \in \mathbf{Z}$; this shows that $L \times L^{\prime} \rightarrow \mathbf{Z}$. For $\alpha \in C \cap(L-M)$, write $\alpha=\sum_{k=0}^{N} u_{k} \xi_{k}$; since $\alpha \notin M$, some $u_{k}$ must be non-integral, say $u_{k^{\prime}}$; we then have $\xi_{k^{\prime}} \in M^{\prime}$ and $\left\langle\alpha, \xi_{k^{\prime}}\right\rangle=u_{k^{\prime}} \notin \mathbf{Z}$. Finally, $G=M^{\prime} / L^{\prime}$ can be identified to the set $[0, p)^{N+1} \cap \mathbf{Z}^{N+1}$. Now all conditions of Theorem 3.4 are satisfied. We thus have (45) and (46). 
Example 3.11. Let $\sigma$ be an $n$-simplex of $\mathbf{R}^{n}$ with the vertex set $\left\{\alpha_{j}\right\}_{j=0}^{n} \subset \mathbf{Z}^{n}$; and let $A$ be the matrix whose columns are the vectors $\left\{\alpha_{j}-\alpha_{0}\right\}_{j=1}^{n}$. Set

$$
\begin{aligned}
a_{j} & =\operatorname{gcd} \text { of the } j \text { th row of adj } B, \\
b_{j} & =\operatorname{gcd} \text { of the } j \text { th row of } B \operatorname{diag}\left[a_{0}, a_{1}, \ldots, a_{n}\right], \\
p_{j} & =\frac{|\operatorname{det} B|}{a_{j}}, \\
q_{j} & =\frac{|\operatorname{det} B|}{\operatorname{gcd}\left(b_{j}, \operatorname{det} B\right)},
\end{aligned}
$$

and $G=\prod_{j=0}^{n} \mathbf{Z} / q_{j} \mathbf{Z}$. Then we have (41) and (42).

Proof. Without loss of generality we assume that det $B$ is positive. Set $V_{1}=V_{2}=\mathbf{R}^{n+1}$, $M_{1}=M_{2}=\mathbf{Z}^{n+1}, L_{1}=\bigoplus_{j=0}^{n} \mathbf{Z} \gamma_{j}, L_{2}=\bigoplus_{j=0}^{n} \mathbf{Z} \gamma_{j}^{\prime}$, where $\gamma_{j}=\beta_{j} / p_{j}, \gamma_{j}^{\prime}=q_{j} e_{j}$. The standard inner product of $\mathbf{R}^{n+1}$ gives a natural pairing $V_{1} \times V_{2} \rightarrow \mathbf{R}$ such that $\left\langle\eta_{j}, \eta_{k}^{\prime}\right\rangle=\delta_{j k}$, where $\eta_{j}=\eta_{j}^{\prime}=e_{j}$. Let $\beta_{j}^{\prime}$ denote the $j$ th row vector of adj $B$ divided by $a_{j}$. The identity $(\operatorname{adj} B) B=(\operatorname{det} B) I_{n}$ shows that $\left\langle\beta_{j}, \beta_{k}^{\prime}\right\rangle=p_{j} \delta_{j k}$. Since both $\beta_{j}$ and $\beta_{j}^{\prime}$ are integral points, $p_{j}$ must be an integer. Then we have conclusions (i) and (ii) of Lemma 3.6. Note that

$$
\left\langle\gamma_{j}, \gamma_{k}^{\prime}\right\rangle=\frac{|\operatorname{det} B|}{p_{j} \operatorname{gcd}\left(b_{k}, \operatorname{det} B\right)}\left\langle\beta_{j}, e_{k}\right\rangle=\frac{a_{j} b_{k j}}{\operatorname{gcd}\left(b_{k}, \operatorname{det} B\right)} \in \mathbf{Z},
$$

where $b_{k j}$ is the $k$ th coordinate of $\beta_{j}$. Then $L_{1} \times L_{2} \rightarrow \mathbf{Z}$.

Now we set $V=V^{\prime}=\mathbf{R}^{n+1}, M=M^{\prime}=\Lambda_{1}, L=L_{1}, L^{\prime}=L_{2}$. Then all conditions of Theorem 3.4 are satisfied, and $G=M^{\prime} / L^{\prime}=\prod_{j=0}^{n} \mathbf{Z} / q_{j} \mathbf{Z}$. We thus have (41) and (42).

As shown in [8], the coefficients of Ehrhart polynomials can be computed from any of such generating functions. In fact, let $L(\sigma, m)=\sum_{j=0}^{n} c_{j}(\sigma) m^{j}$. Then

$$
\begin{aligned}
\sum_{m=0}^{\infty} L(\sigma, m) e^{-2 \pi m s} & =\sum_{m=0}^{\infty} \sum_{j=0}^{n} c_{j}(\sigma) m^{j} e^{-2 \pi m s} \\
& =\sum_{j=0}^{n} \frac{c_{j}(\sigma)}{(-2 \pi)^{j}} \sum_{m=0}^{\infty} \frac{d^{j}}{d s^{j}} e^{-2 \pi m s} \\
& =\sum_{j=0}^{n} \frac{c_{j}(\sigma)}{(-2 \pi)^{j}} \cdot \frac{d^{j}}{d s^{j}}\left(\frac{1}{1-e^{-2 \pi s}}\right) \\
& =\sum_{j=0}^{n} \frac{c_{j}(\sigma)}{(-2 \pi)^{j}} \cdot \frac{d^{j}}{d s^{j}}\left(\frac{1}{2 \pi s}+\cdots\right) \\
& =\sum_{j=0}^{n} \frac{j ! c_{j}(\sigma)}{(2 \pi)^{j+1}}\left(\frac{1}{s^{j+1}}+\cdots\right),
\end{aligned}
$$


where the dots $\cdots$ mean the holomorphic terms near $s=0$. So we have the following proposition on the coefficients of Ehrhart polynomials in the generating functions.

Proposition 3.12. The $j$ th coefficient of the Ehrhart polynomial $L(\sigma, m)$ is the coefficient of the term $1 / s^{j+1}$ in the Laurent expansion at $s=0$ of the function

$$
\frac{(2 \pi)^{j+1}}{j !} \sum_{m=0}^{\infty} L(\sigma, m) e^{-2 \pi m s}
$$

\section{Applications and Dedekind Sums}

It is not surprising from (29) and (30) that the coefficients of Ehrhart polynomials are related to the function $((t))$, defined by

$$
((t))= \begin{cases}t-\lceil t\rceil+\frac{1}{2} & \text { for } t \notin \mathbf{Z}, \\ 0 & \text { for } t \in \mathbf{Z},\end{cases}
$$

thereby related to Dedekind sums. Recall the Dedekind $\operatorname{sum} s(q, p)$ of coprime positive integers $p$ and $q$ is defined by

$$
s(q, p)=\sum_{k=1}^{p-1}\left(\left(\frac{q k}{p}\right)\right)\left(\left(\frac{k}{p}\right)\right) .
$$

Since $((t))$ is a periodic odd function of period 1 , it is routine to check the following properties (see [19]):

$$
\begin{aligned}
\sum_{k=0}^{p-1}\left(\left(\frac{k+t}{p}\right)\right) & =((t)) \\
\sum_{k=0}^{p-1}\left(\left(\frac{k}{p}\right)\right)^{l} & =0 \quad \text { for odd } l \\
\sum_{k=0}^{p-1}\left(\left(\frac{k}{p}\right)\right)^{2} & =\frac{p}{12}+\frac{1}{6 p}-\frac{1}{4}
\end{aligned}
$$

Let $\sigma\left(a_{1}, \ldots, a_{n}\right)$ be the lattice $n$-simplex of $\mathbf{R}^{n}$ whose vertices are $\left(0, \ldots, 0, a_{j}, 0\right.$, $\ldots, 0)$ and the origin; $a_{j}$ are positive integers. We apply (29) to compute explicitly the coefficient $c_{n-2}$ of the Ehrhart polynomial $L\left(\sigma^{0}\left(a_{1}, \ldots, a_{n}\right), t\right)$. For simplicity we assume that $a_{j}$ are pairwise coprime and write $a=a_{1} a_{2} \cdots a_{n}$. From (29), $c_{n-2}$ can be written as

$$
c_{n-2}=\frac{1}{n !} \sum_{j_{1}=0}^{a_{1}-1} \cdots \sum_{j_{n}=0}^{a_{n}-1} s_{2}\left(\left\lceil\frac{j_{1}}{a_{1}}+\cdots+\frac{j_{n}}{a_{n}}\right\rceil-1, \cdots,\left\lceil\frac{j_{1}}{a_{1}}+\cdots+\frac{j_{n}}{a_{n}}\right\rceil-n\right) .
$$

If $j_{1} / a_{1}+\cdots+j_{n} / a_{n}=k$ is an integer, then $\left(a / a_{1}\right) j_{1}+\cdots+\left(a / a_{n}\right) j_{n}=k a$ implies $j_{i} \equiv 0\left(\bmod a_{i}\right)$ for all $1 \leq i \leq n$; so $j_{1}=\cdots=j_{n}=0$. Thus if 
$\left(j_{1}, \ldots, j_{n}\right) \neq(0, \ldots, 0)$,

$$
\left\lceil\frac{j_{1}}{a_{1}}+\cdots+\frac{j_{n}}{a_{n}}\right\rceil=\left(\frac{j_{1}}{a_{1}}+\cdots+\frac{j_{n}}{a_{n}}\right)-\left(\left(\frac{j_{1}}{a_{1}}+\cdots+\frac{j_{n}}{a_{n}}\right)\right)+\frac{1}{2} .
$$

Write $u=j_{1} / a_{1}+\cdots+j_{n} / a_{n}$. For positive integers $1 \leq k<l \leq n$, we have

$$
\begin{aligned}
f(u):= & \sum_{k<l}\left(u-((u))-k+\frac{1}{2}\right)\left(u-((u))-l+\frac{1}{2}\right) \\
= & \sum_{k<l}\left(u^{2}+((u))^{2}-2 u((u))\right)-\left(\sum_{k<l}(k+l-1)\right)(u-((u))) \\
& +\sum_{k<l}\left(k l-\frac{k+l}{2}+\frac{1}{4}\right) .
\end{aligned}
$$

We understand that the summation $\sum_{j_{1}, \ldots, j_{n}} f(u)$ is taken over $0 \leq j_{1} \leq a_{1}-1, \ldots, 0 \leq$ $j_{n} \leq a_{n}-1$ and so forth in the following. Notice the difference between $c_{n-2}$ and $\sum_{j_{1}, \ldots, j_{n}} f(u)$ at $j_{1}=\cdots=j_{n}=0$. It follows that

$$
c_{n-2}=\frac{1}{n !}\left[\sum_{j_{1}, \ldots, j_{n}} f(u)+s_{2}(-1, \ldots,-n)-\sum_{k<l}\left(k l-\frac{k+l}{2}+\frac{1}{4}\right)\right] .
$$

An elementary careful calculation shows that

$$
\begin{aligned}
\sum_{j_{1}, \ldots, j_{n}} u^{2} & =\frac{n(3 n+1) a}{12}+\frac{a}{2} \sum_{k=1}^{n}\left(-\frac{n}{a_{k}}+\frac{1}{3 a_{k}^{2}}\right)+\frac{a}{2} \sum_{k<l} \frac{1}{a_{k} a_{l}} ; \\
\sum_{j_{1}, \ldots, j_{n}}((u))^{2} & =\frac{a}{12}+\frac{1}{6 a}-\frac{1}{4} ; \\
\sum_{j_{1}, \ldots, j_{n}} u((u)) & =\sum_{k=1}^{n} s\left(\frac{a}{a_{k}}, a_{k}\right) ; \\
\sum_{j_{1}, \ldots, j_{n}} u & =\frac{a}{2}\left(n-\sum_{k=1}^{n} \frac{1}{a_{k}}\right) ; \\
\sum_{k<l}(k+l-1) & =\frac{(n-1) n^{2}}{2} ; \\
\sum_{k<l}\left(k l-\frac{k+l}{2}+\frac{1}{4}\right) & =\frac{(n-1) n\left(3 n^{2}-n-1\right)}{24} \\
s_{2}(-1, \ldots,-n) & =\frac{(n-1) n(n+1)(3 n+2)}{24} .
\end{aligned}
$$

Proposition 4.1. Let $a_{1}, \ldots, a_{n}$ be pairwise coprime positive integers. Set $a=a_{1} a_{2}$ $\cdots a_{n}$. Then the coefficient $c_{n-2}$ of the Ehrhart polynomial $L\left(\sigma^{0}\left(a_{1}, \ldots, a_{n}\right), t\right)$ is 
given by

$$
c_{n-2}=\frac{1}{(n-2) !}\left[\frac{n}{4}+\frac{1}{12 a}+\sum_{j=1}^{n}\left(\frac{a}{12 a_{j}^{2}}-s\left(\frac{a}{a_{j}}, a_{j}\right)\right)+\sum_{1 \leq k<l \leq n} \frac{a}{4 a_{k} a_{l}}\right] .
$$

Take $n=2$, then $c_{0}$ is the Euler characteristic of a relatively open triangle so that $c_{0}=1$. We thus obtain the following well-known relation on Dedekind sums; see [19].

Corollary 4.2 (Reciprocity Law of Dedekind Sums). For any coprime positive integers $a_{1}$ and $a_{2}$,

$$
s\left(a_{1}, a_{2}\right)+s\left(a_{2}, a_{1}\right)=-\frac{1}{4}+\frac{1}{12}\left(\frac{1}{a_{1} a_{2}}+\frac{a_{1}}{a_{2}}+\frac{a_{2}}{a_{1}}\right) .
$$

Take $n=3$, then we have the coefficient $c_{1}$ for the Ehrhart polynomial of the closed tetrahedron with vertices $(0,0,0),\left(a_{1}, 0,0\right),\left(0, a_{2}, 0\right),\left(0,0, a_{3}\right)$, where $a_{1}, a_{2}, a_{3}$ are pairwise coprime, as given by

$$
\begin{aligned}
c_{1}= & \frac{3}{4}+\frac{1}{12}\left(\frac{1}{a_{1} a_{2} a_{3}}+\frac{a_{1} a_{2}}{a_{3}}+\frac{a_{1} a_{3}}{a_{2}}+\frac{a_{2} a_{3}}{a_{1}}\right)+\frac{1}{4}\left(a_{1}+a_{2}+a_{3}\right) \\
& -s\left(a_{1} a_{2}, a_{3}\right)-s\left(a_{1} a_{3}, a_{2}\right)-s\left(a_{2} a_{3}, a_{1}\right) .
\end{aligned}
$$

Adding up the coefficients $c_{0}=1, c_{1}, c_{2}=\frac{1}{4}\left(a_{1} a_{2}+a_{1} a_{3}+a_{2} a_{3}+1\right)$, and $c_{3}=$ $a_{1} a_{2} a_{3} / 6$, one obtains Mordell's formula [15] on the number of lattice points of the closed tetrahedron.

The other coefficients of $L\left(\sigma\left(a_{1}, \ldots, a_{n}\right), t\right)$ can be calculated in a similar way, using Zagier's higher-dimensional Dedekind sums [10], [21], but the calculation is too complicated to be presented here. However, it is not clear whether the coefficients of the Ehrhart polynomial for an arbitrary lattice simplex can be fully expressed in terms of the higher-dimensional Dedekind sums. Since $c_{0}=(-1)^{n}$ for any relatively open $n$-simplex, it reduces to the reciprocity law for higher-dimensional Dedekind sums. In order to express fully the coefficients of Ehrhart polynomials in terms of certain cotangent expansions, it seems that one needs to generalize the Dedekind sums further. We introduce a matrix Dedekind sum in the following.

Definition 4.3. Let $A$ be an $m \times n$ integral matrix and let $B$ be an $l \times n$ integral matrix whose row vectors are denoted $\beta_{1}, \ldots, \beta_{l}$. Let $p=\left(p_{1}, \ldots, p_{m}\right), q=\left(q_{1}, \ldots, q_{n}\right)$, and $r=\left(r_{1}, \ldots, r_{l}\right)$ be integral vectors with positive entries. The matrix Dedekind sum is

$$
s(A, B ; p, q, r)=\sum_{\substack{0 \leq h<q \\ h \mathbb{Z}^{n} \\ p \mid A h}} \prod_{j=1}^{l}\left(\left(\frac{\beta_{j} h}{r_{j}}\right)\right),
$$

where $0 \leq h<q$ means that all the entries of $h$ are non-negative and strictly less than the corresponding entries of $q ; p \mid A h$ means that each entry of $p$ divides the corresponding entry of $A h$. 
The idea to define the matrix Dedekind sum (49) comes from formulas (29) and (50) for coefficients $c_{j}$ and the calculation of $c_{n-2}$. Theorem 2.6 is also important to imagine what sum should be taken over. The right side of (29) can be divided into two parts: Part 1, a sum over $|\gamma| \notin \mathbf{Z}$; and Part 2, a sum over $|\gamma| \in \mathbf{Z}$. In Part 1, since $\lceil|\gamma|\rceil=|\gamma|-((|\gamma|))+\frac{1}{2}$, the sum can be expanded into a collection of sums

$$
\sum a(k, l)|\gamma|^{k}((|\gamma|))^{l}, \quad k+l \leq n-j .
$$

These sums can be further reduced to a collection of sums of the form (49), some of them may be in rational forms without involving the symbol $((\cdot))$. In Part 2, the sum may be written as counting the number of lattice points of some lower-dimensional lattice polytopes. By induction on dimension, they can be reduced in principle to a collection of sums of the form (49). However, one may still doubt the program with (49) until it is actually done explicitly. Since $c_{0}\left(\sigma^{0}\right)=(-1)^{n}$ for any lattice $n$-simplex $\sigma$, it is sure that the program will give certain reciprocity laws on certain generalized Dedekind sums (may not be discovered yet) when the right side of (29) is treated correctly in arithmetic ways. Study of this matrix Dedekind sum in the special case $p_{1}=\cdots=p_{m}=r_{1}=\cdots=r_{l}$ is particularly wanted.

Theorem 4.4. Let $\sigma$ be an n-simplex of $\mathbf{R}^{N}$ with the vertex set $\left\{\alpha_{j}\right\}_{j=0}^{n} \subset \mathbf{Z}^{N}$; and let $A$ be the $N \times n$ matrix whose columns are the vectors $\left\{\alpha_{j}-\alpha_{0}\right\}_{j=0}^{n}$. Let $a_{j}$ be the gcd of the $j$ th row of $\operatorname{adj}\left(A^{\mathrm{T}} A\right) A^{\mathrm{T}} ; p_{j}=\left|\operatorname{det} A^{\mathrm{T}} A\right| / a_{j}$. If $p$ is a positive integer such that either $\operatorname{lcm}\left\{p_{j}\right\}_{j=0}^{n} \mid p$ or $(\operatorname{tor} A) \mid p$, then the $j$ th coefficient of the Ehrhart polynomial $L\left(\sigma^{0}, t\right)$ is given by

$$
c_{j}\left(\sigma^{0}\right)=\frac{1}{n !} \sum_{\substack{h_{1}, \ldots, h_{n}=0 \\ h\left(h_{1}, \ldots, h_{n}\right) \\ p \mid A h}}^{p-1} s_{n-j}\left(\left\lceil\frac{h_{1}+\cdots+h_{n}}{p}\right\rceil-1, \cdots,\left\lceil\frac{h_{1}+\cdots+h_{n}}{p}\right\rceil-n\right),
$$

where $p \mid$ Ah means that $p$ divides each entry of the integral vector Ah.

Proof. This is an immediate consequence of Theorm 2.6 and the coefficient formula (29).

Formula (50) shows directly that $c_{j}\left(\sigma^{0}\right)$ is invariant under unimodular $N \times N$ matrices. Write $N=m$ and $\operatorname{dim} \sigma=n$; expanding the symmetric functions in the right side of (50) and using $c_{0}\left(\sigma^{0}\right)=(-1)^{\operatorname{dim} \sigma}$, we have the following corollary.

Corollary 4.5 (Reciprocity Law). Let $A$ be an $m \times n$ integral matrix with $\operatorname{rank} A=n$ and $p=$ tor $A$. Then

$$
\sum_{j=0}^{n}(-1)^{j} s_{n-j}(1,2, \ldots, n) \sum_{\substack{h_{1}, \ldots, h_{n}=0 \\ h=\left(h_{1} \ldots h_{n}\right) \\ p \mid A h}}^{p-1}\left\lceil\frac{h_{1}+\cdots+h_{n}}{p}\right\rceil^{j}=n !
$$

Let $P$ be a bounded lattice polyhedron of $\mathbf{R}^{N}$ whose vertices are points of the integral lattice $\mathbf{Z}^{N}$, decomposed into a collection $\mathcal{F}$ of disjoint relatively open lattice simplices. 
The subcollection of $n$-simplices are denoted $\mathcal{F}(n)$. Then the $j$ th coefficient of the Ehrhart polynomial $L(P, t)$ can be written as

$$
c_{j}(P)=\sum_{\alpha \in \mathcal{F}(0)} \sum_{n=0}^{\operatorname{dim} P} \frac{1}{(n+1) !} \sum_{\substack{\alpha \leq \sigma \in \mathcal{F}(n) \\ \gamma \in D(\sigma, \alpha)}} s_{n-j}(\lceil|\gamma|\rceil-1, \ldots,\lceil|\gamma|\rceil-n) .
$$

\section{Acknowledgments}

I thank Victor Klee and Richard Stanley for their hospitality during my visit at the University of Washington and MIT, where this work was done. Especially, I thank Stephen Schanuel for his suggestion of using a torsion subgroup to define the torsion for an Abelian group homomorphism. Finally, I thank the referees for carefully reading the manuscript and offering some important comments.

\section{References}

1. A. I. Barvinok, Computing the Ehrhart polynomial of a convex lattice polytope, Discrete Comput. Geom. 12 (1994), 35-48.

2. M. Brion and M. Vergne, Lattice points in simple polytopes, J. Amer. Math. Soc. 10 (1997), 371-392.

3. S. E. Cappell and J. L. Shaneson, Genera of algebraic varieties and counting lattice points, Bull. Amer. Math. Soc. 30 (1994), 62-69.

4. B. Chen, On the Euler characteristics of finite unions of convex sets, Discrete Comput. Geom. 10 (1993), 79-93.

5. B. Chen, Weight functions, double reciprocity laws, and volume formulas for lattice polyhedra, Proc. Nat. Acad. Sci. USA, 95 (1998), 9093-9098.

6. B. Chen and V. Tauraev, Counting lattice points of rational polyhedra, Adv. in Math. 155 (2000), 84-97.

7. V. I. Danilov, The geometry of toric varieties, Russian Math. Surveys 33 (1978), 97-154.

8. R. Diaz and S. Robins, The Ehrhart polynomial of a lattice polytope, Ann. of Math. 135 (1997), 503-518.

9. E. Ehrhart, Sur un problème de geómétrie diophantienne linéaire II, J. Reine Angew. Math. 227 (1967), $25-49$.

10. F. Hirzebruch and D. Zagier, The Atiyah-Singer Index Theorem and Elementary Number Theory, Publish or Perish, Boston, MA, 1974.

11. J. M. Kantor and A. G. Khovanskii, Une application du Théoréme de Riemann-Roch combinatorie au polynôme d'Ehrhart des polytopes entiers de $\mathbf{R}^{n}$, C. R. Acad. Sci. Paris, Sér. I 317 (1993), 501-507.

12. D. A. Klain and G.-C. Rota, Introduction to Geometric Probability, Cambridge University Press, Cambridge, 1997.

13. I. G. Macdonald, The volume of a lattice polyhedron, Math. Proc. Cambridge Philos. Soc. 59 (1963), 719-726.

14. I. G. Macdonald, Polynomials associated with finite cell complexes, J. London Math. Soc. (2) 4 (1971), 181-192.

15. L. J. Mordell, Lattice points in a tetrahedron and Dedekind sums, J. Indian Math. 15 (1951), 41-46.

16. R. Morelli, Pick's theorem and the Todd class of toric variety, Adv. in Math. 100 (1993), 183-231.

17. M. Newmans, Integral Matrices, Pure and Applied Mathematics, vol. 45, Academic Press, New York, 1972.

18. J. Pommersheim, Toric varieties, lattice points and Dedekind sums, Math. Ann. 295 (1993), 1-24.

19. H. Rademacher and E. Grosswald, Dedekind Sums, The Carus Mathematical Monographs, vol. 16, The Mathematical Association of America, Washington, DC, 1972.

20. R. P. Stanley, Enumerative Combinatorics, Vol. I, Cambridge University Press, Cambridge, 1997.

21. D. Zagier, Higher dimensional Dedekind sums, Math. Ann. 202 (1973), 149-172.

Received May 18, 2000, and in revised form March 3, 2002. Online publication July 24, 2002. 